Please do not remove this page

RMIT

UNIVERSITY

\title{
Efficient computation of iceberg cubes by bounding aggregate functions
}

Zhang, Xiuzhen; Chou, Pauline; Dong, Guozhu

https://researchrepository.rmit.edu.au/esploro/outputs/9921858737301341/filesAndLinks?institution=61RMIT_INST\&index=null

Zhang, X., Chou, P., \& Dong, G. (2007). Efficient computation of iceberg cubes by bounding aggregate functions. IEEE Transactions On Knowledge And Data Engineering, 19, 903-918.

https://doi.org/10.1109/TKDE.2007.1053

Published Version: https://doi.org/10.1109/TKDE.2007.1053

Repository homepage: https://researchrepository.rmit.edu.au

(c) 2007 IEEE. Personal use of this material is permitted. However, permission to reprint/republish this material for advertising or promotional purposes or for creating new collective works for resale or redistribution to servers or lists, or to reuse any copyrighted component of this work in other works must be obtained from the IEEE.

Downloaded On 2023/04/26 19:42:27 +1000 


\title{
Efficient Computation of Iceberg Cubes by Bounding Aggregate Functions
}

\author{
Xiuzhen Zhang, Pauline Lienhua Chou, and Guozhu Dong, Senior Member, IEEE
}

\begin{abstract}
The iceberg cubing problem is to compute the multidimensional group-by partitions that satisfy given aggregation constraints. Pruning unproductive computation for iceberg cubing when nonantimonotone constraints are present is a great challenge because the aggregate functions do not increase or decrease monotonically along the subset relationship between partitions. In this paper, we propose a novel bound prune cubing (BP-Cubing) approach for iceberg cubing with nonantimonotone aggregation constraints. Given a cube over $n$ dimensions, an aggregate for any group-by partition can be computed from aggregates for the most specific $n$-dimensional partitions (MSPs). The largest and smallest aggregate values computed this way become the bounds for all partitions in the cube. We provide efficient methods to compute tight bounds for base aggregate functions and, more interestingly, arithmetic expressions thereof, from bounds of aggregates over the MSPs. Our methods produce tighter bounds than those obtained by previous approaches. We present iceberg cubing algorithms that combine bounding with efficient aggregation strategies. Our experiments on real-world and artificial benchmark data sets demonstrate that BP-Cubing algorithms achieve more effective pruning and are several times faster than state-of-the-art iceberg cubing algorithms and that BP-Cubing achieves the best performance with the top-down cubing approach.
\end{abstract}

Index Terms-Data mining, data cube, pruning, data warehouses.

\section{INTRODUCTION}

$W^{\prime}$ ITH the multidimensional model for data warehouses, a data set consists of tuples over dimensions and measures, and queries involve aggregating the measures over partitions of tuples sharing identical dimension values. For example, the Structured Query Language (SQL) in Fig. 1a gives "the monthly total amount of sales for each city" on the Sales data set in Table 1. The (Month, City) group-by divides the data set into partitions of tuples sharing identical (Month, City) values, and the measure Sale is aggregated to yield Sum ( $\mathrm{Sale}$ ) for each partition. The Cube operator was proposed [6] to compute all potential group-bys of a data set, leading to the notion of a data cube.

The iceberg cube was proposed in [4], where only partitions whose aggregate values satisfy an aggregation constraint are produced. By adding "HAVING $\operatorname{Count}(*) \geq 10^{\prime \prime}$ to the SQL query in Fig. 1a, we get an SQL query (in Fig. 1b) for computing those (Month, City) partitions, each having $\geq 10$ tuples. The aggregation constraint $\operatorname{Count}(*) \geq 10$ is antimonotone: If a partition does not contain $\geq 10$ tuples and thus fails the constraint, then all its subpartitions will have fewer tuples and also fail the constraint. The bottom-up cubing (BUC) strategy was proposed [4], where aggregates are computed starting from

- X. Zhang and P.L. Chou are with the School of Computer Science and IT, RMIT University, GPO Box 2476v, Melbourne 3001, Australia. E-mail: $\{$ zhang, lchou\}@cs.rmit.edu.au.

- G. Dong is with the Department of Computer Science and Engineering, Wright State University, Dayton, $\mathrm{OH} 45435$.

E-mail: guozhu.dong@wright.edu.

Manuscript received 18 Sept. 2005; revised 12 Apr. 2006; accepted 15 Jan. 2007; published online 25 Apr. 2007.

For information on obtaining reprints of this article, please send e-mail to: tkde@computer.org, and reference IEEECS Log Number TKDE-0385-0905.

Digital Object Identifier no. 10.1109/TKDE.2007.1053. the partition of all tuples and then followed recursively with the subpartitions, and antimonotone constraints are used for pruning.

In On-Line Analytical Processing (OLAP) applications, aggregation constraints often involve complex aggregates. The constraint $\operatorname{Sum}(x) \geq 5,000$ and $\operatorname{Var}(x) \leq 100$, which states that "the total profit $(x)$ is at least $\$ 5,000$, and the variance of profit is at most $\$ 100, "$ is a typical example of constraints for supporting enterprise data analysis and decision making. As $x$ can be positive or negative, the subset relationship between a group ${ }^{1}$ and its subgroups may not imply monotonically increasing (or decreasing) aggregate values. Computing iceberg cubes with such constraints has been a challenging problem. Existing proposals in the literature have all followed the approach of deriving weaker but antimonotone constraints [7], [15]. They are restricted in two aspects: 1 ) the weaker constraints derived from nonantimonotone constraints are often very loose and not effective enough for pruning, especially for complex constraints, and 2) aggregates are computed following the BUC framework, and only one group is aggregated at a time in the recursive partitioning process, which limits the amount of information readily available for pruning.

In this paper, we propose a novel technique, called bound prune cubing (BP-Cubing), for efficiently computing iceberg cubes with nonantimonotone aggregation constraints. The main ideas are to effectively derive tight bounds for possible aggregates and to use such bounds to prune unproductive computation. An important part is played by the "most specific partitions" (MSPs), namely, those nonempty partitions that cannot be further divided (for the subcube under consideration). MSPs can be viewed as the

1. We use group and partition as synonyms. 


$\begin{array}{ll}\text { SELECT Month, City, Sum(Sale) } & \text { SELECT Month, City, Sum(Sale) } \\ \text { FROM Sales } & \text { FROM Sales } \\ \text { GROUP BY Month, City } & \text { GROUP BY Month, City } \\ & \text { HAVING Count }(*) \geq 10\end{array}$

(a)

(b)

Fig. 1. Two SQL group-by queries on the sales data set. (a) An SQL group-by. (b) Adding a constraint to (a).

TABLE 1

A Sales Data Set, Partially Aggregated

\begin{tabular}{llllll}
\hline Month & Product & SalesMan & City & Sum(Sale) & Count $\left(^{*}\right)$ \\
\hline January & Toy & John & Perth & 200 & 5 \\
March & TV & Peter & Perth & 100 & 40 \\
March & TV & John & Perth & 100 & 20 \\
March & TV & John & Sydney & 100 & 10 \\
April & TV & Peter & Perth & 100 & 8 \\
April & Toy & Peter & Sydney & 100 & 5 \\
\hline
\end{tabular}

Month, Product, SalesMan, and City are dimensions. Sale is the measure.

basic units for computing data cubes, since other partitions are unions of some MSPs. The aggregate for any partition in the data cube can be computed from aggregating some MSPs. Decomposing an aggregate function into the base aggregate functions Count, Max, Min, and Sum on MSPs, the aggregate function can be tightly bounded. By using MSPs, nonexisting groupings of tuples will not influence the estimation of the bounds of aggregates, ${ }^{2}$ which is another reason why tight bounds can be obtained.

With a four-dimensional data cube on the average Sale (Avg (Sale) ) of Sales, six MSPs are shown in Table 1. For each MSP, $\operatorname{Avg}(\operatorname{Sale})=\operatorname{Sum}(\operatorname{Sale}) / \operatorname{Count}(*){ }^{3}$ We now illustrate cases where $\mathrm{Sum}(\mathrm{Sale})>0$ for each MSP (the general case is treated later). Both Sum(Sale) and Count $(*)$ increase monotonically over unions of "positive" MSPs. The upper bound of Avg (Sale) can be obtained from the upper bound for Sum (Sale) and the lower bound for $\operatorname{Count}(*)$, which is $\operatorname{Sum}_{\mathrm{i}}(\operatorname{Sum}(\operatorname{Sale})) / \operatorname{Min}_{\mathrm{i}}(\operatorname{Count}(*))$, where $i$ ranges over the six MSPs. This can, in fact, be improved: The upper bound for Avg (Sale) is actually $\operatorname{Max}_{\mathrm{i}} \operatorname{Avg}(\mathrm{Sale})$, where $i$ ranges over all MSPs (see Section 6). For Table 1, $\operatorname{Max}_{\mathrm{i}} \operatorname{Avg}(\mathrm{Sale})$ is $200 / 5=40$, reached at the group (January, Toy, John, Perth).

The MSPs can be obtained by a single scan of the raw data. The bound-prune technique described above will be applied recursively for all subcubes. We will optimize the computation for subcubes by reusing computed results for supercubes.

Our bound-prune technique can be applied in the BUC approach. More importantly, it also fits nicely into the topdown cubing approach, where multiway shared computation of aggregates improves cubing efficiency. Bound-prune is a general technique that can prune for constraints of the form " $F(x) \geq \alpha$," " $F(x) \leq \alpha$," or " $F(x)$ in $\left[\alpha_{1}, \alpha_{2}\right]$. " The aggregate function $F$ can be an SQL aggregate function or a commonly seen complex aggregate function such as Average (Avg) or Variance (Var). As will be shown later

2. This is an issue for other approaches.

3. For ease of discussion, we assume that NULL is not allowed for measures and, therefore, Count $($ Sale $)=\operatorname{Count}(*)$.

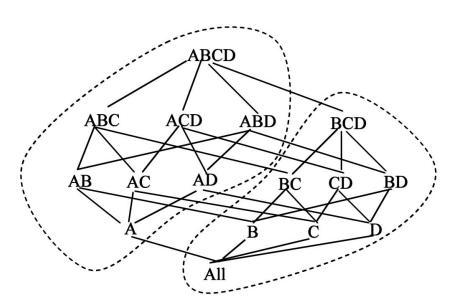

(a)

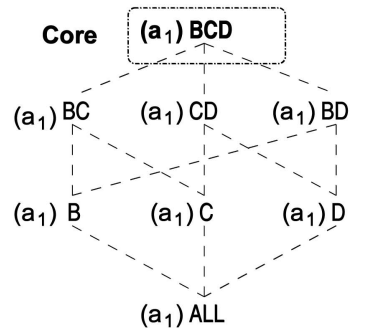

(b)
Fig. 2. Data cube lattice and subdata cube. (a) Cube ( $A B C D$ ) decomposition. (b) Cube (BCD) $\mid a_{1}$.

in our experiments on real-world and synthetic benchmark data sets, our BP-Cubing algorithms are several times faster than existing pruning techniques, including the most recent Divide-and-Approximate (DnA) algorithm [15].

Organizationally, Section 2 gives some preliminaries. Section 3 defines boundability of aggregate functions. We discuss how we can derive the bounds for base aggregates, their functions, and complex aggregates in Sections 4, 5, and 6 , respectively. Section 7 then presents the BP-Cubing algorithms. We report experimental evaluations in Section 8. We discuss related works in Section 9 and conclude in Section 10.

\section{Some Preliminaries on Data Cubes}

We will use uppercase letters to denote dimensions and lowercase letters to denote dimension values. A group-by is a tuple of dimensions of the form $(A, B, C)$, and a partition of $(A, B, C)$ is a tuple of dimension values of the form $(a, b, c)$.

The group-bys in a data cube form a lattice structure called the cube lattice. Fig. 2 shows an example fourdimensional cube lattice. The empty group-by (which aggregates all tuples in a data set) is at the bottom of the lattice, and the group-by with all dimensions is at the top. The edges in the lattice represent subset-superset relationships between group-bys.

A special value " $*$ " is used in specifying partitions, with the meaning that it can match any value (of the applicable dimension). For the data set in Table 1, (Jan, $*, *, *)$ denotes the partition of tuples having Month $=$ January and having no restriction on the other dimensions. For brevity, " $*$ " is usually omitted in naming partitions; for example, $(\operatorname{Jan}, *, *, *)$ is written as ( $\operatorname{Jan})$.

The subset relationship also exists between partitions from different group-bys. For example, the 3D partition (Jan, TV, Perth) is a subset of each of the 2D partitions (Jan, TV), (Jan, Perth), and (TV, Perth).

Given a data set $S$ of $n$ dimensions $A_{1}, \ldots, A_{n}$ and a measure $X$, the corresponding data cube is usually referred to as $\operatorname{Cube}\left(A_{1}, \ldots, A_{n}\right)$. Here, we think of the cube as the set of possible group-bys or the set of possible groups for all group-bys. For example, the four-dimensional cube in Table 1 can be denoted as Cube (Month, Product, Salesman, City).

For ease of discussion, we also refer to the cube as $\operatorname{Cube}(X)$, and think of it as the "measure partitions" (the possible bags of measure values for the possible groups). 
Specifically, let $g_{1}, \ldots, g_{m}$ be all possible groups of the cube. By an abuse of notation, we will use $X_{i}$ to denote the bag (multiset) $\left\{t[X] \mid t \in g_{i}\right\}$, and we will refer to $X_{1}, \ldots, X_{m}$ as the measure partitions. Now, given an aggregate function $F$, we apply $F$ to the measure partitions in the data cube to get $F(\operatorname{Cube}(X))=\left\{F\left(X_{i}\right) \mid X_{i} \in C u b e(X)\right\} .{ }^{4}$ With the aggregate function Sum (Sale), we can write Sum(Cube (Sale)) for the four-dimensional cube in Table 1.

Aggregate functions are categorized into distributive, algebraic, and holistic functions, depending on how an aggregate on a partition can be computed from aggregates on its subpartitions [6]. 1) An aggregate function $F$ is distributive if there is a function $G$ such that $F(S)=$ $G\left(\left\{F\left(S_{i}\right) \mid i=1, \ldots, n\right\}\right)$ for a data set $S$ and partitioning $\left\{S_{1}, \ldots, S_{n}\right\}$ of $S .{ }^{5}$ For example, Sum and Max are distributive, with $G=F$, and Count is distributive, with $G=$ Sum. 2) An aggregate function $F$ is algebraic if it can be computed by a function $H$ with several arguments, each of which is obtained by applying a distributive aggregate function. Avg, Var, Standard-Deviation, and MaxN and $\mathrm{MinN}^{6}$ are algebraic functions; for example, Avg is algebraic, since $\operatorname{Avg}(S)=\operatorname{Sum}(S) / \operatorname{Count}(S)$, and both Sum and Count are distributive. All distributive aggregations are algebraic. 3) An aggregate function $F$ is holistic if it is not distributive or algebraic. Rank, Mode, and Median are examples of holistic functions. We will call the distributive aggregations used for computing algebraic functions $F$ (item 2 above) the auxiliary aggregates.

Existing cubing algorithms have made use of the properties of distributive and algebraic functions to compute supergroups from subgroups [1], [10], [11], [17]. No efficient cubing algorithms for holistic functions have been reported. It is worth noting that in most applications, aggregate functions are algebraic functions. In the discussions below, the term "aggregate functions" will refer to distributive and algebraic functions unless specified otherwise.

\section{Bounding Data Cubes}

The basic idea of bounding is to estimate the upper and lower bounds for a cube from the smallest or the MSPs of the base table. In this section, we define the boundability concept for aggregate functions. In Sections 4 and 5, we will discuss how we can bound the base aggregate functions Count, Max, Min, and Sum and how we can bound algebraic functions given as arithmetic expressions of the base aggregate functions.

Definition 1 (most specific partition and data cube core). Given a data set on dimensions $A_{1}, \ldots, A_{n}$ and measure $X$, all $n$-dimensional partitions form the core of the data cube:

$$
\left\{\left(a_{1}, \ldots, a_{n}\right) \mid a_{i} \in \operatorname{domain}\left(A_{i}\right), a_{i} \neq{ }^{\prime \prime} *^{\prime \prime}, 1 \leq i \leq n\right\} .
$$

Each partition in the core is an MSP. The multiset of measure values for tuples in an MSP $g$, namely, $\{t[X] \mid t \in g\}$, is an MSP of the measure.

All aggregates in a data cube can be computed from its MSPs. For the Sales data set in Table 1 and Cube (Month,

4. Strictly speaking, $F\left(X_{i}\right)$ is the $F$ aggregate of $X$ for group $g_{i}$.

5. $S=\cup_{i=1}^{n} S_{i}$ and $S_{i} \cap S_{j}=\phi$ for $i \neq j$.

6. MaxN and MinN return, respectively, the $N$ largest and smallest values.
Product, SalesMan, City), the core consists of six (Month, Product, Salesman, City) partitions, namely, (Jan, Toy, John, Perth), (Mar, TV, Peter, Perth), (Mar, TV, John, Perth), (Mar, TV, John, Sydney), (Apr, TV, Peter, Perth), and (Apr, Toy, Peter, Sydney). Any aggregate in this cube can be computed from a subset of the six MSPs. We thus can use MSPs to bound the aggregates in a data cube.

Definition 2 (Aggregate function bound). An upper bound of an aggregate function $F$ for the data cube on $X$ is a real number $U$ such that for any partition $X_{i}$ of $\operatorname{Cube}(X)$, it is the case that $F\left(X_{i}\right) \leq U$. Similarly, we can define a lower bound for $F(\operatorname{Cube}(X))$ to be a real number $L$ such that for any partition $X_{i}$ of $\operatorname{Cube}(X), F\left(X_{i}\right) \geq L$.

Observation 1. Given a data cube on measure $X$ and an aggregate function $F$, the tightest upper bound and lower bound are, respectively, reached by the largest and smallest aggregate values that can be produced by any set of MSPs of the data cube.

Example 1. In Table 1, Sum (Sale) for any group in the data cube is not larger than the sum of Sum (Sale) for all six MSPs, which is 700. On the other hand, Sum(Sale) for any group in the data cube is not smaller than the minimal Sum (Sale) among the six MSPs, which is 100. Therefore, for Sum(Sale), Cube (Month, Product, SalesMan, City) has the tightest upper bound of 700 and the tightest lower bound of 100 .

The notion of MSP and data cube core is also applied to subdata cubes. Fig. 2a shows Cube $(A B C D)$, and its MSPs are the ABCD partitions. The polygon on the left of Fig. 2a denotes a set of lattice structures, as shown in Fig. 2b. The polygon on the right of Fig. 2a represents Cube (BCD). The MSPs for Cube (BCD) are BCD partitions, which are unions of $A B C D$ partitions, with equal values for $B, C$, and $D$. The lattice structure, as shown in Fig. $2 b$, is called a subdata cube. For the lattice in Fig. 2b, $\left(a_{1}, \mathrm{~B}, \mathrm{C}, \mathrm{D}\right)$ partitions, which are a subset of the ABCD partitions, form its core. The relationship between cores for a cube and its subcubes, and that for a cube and its lower-dimensional counterparts, are important for computing bounds from MSPs, without incurring extra cost and for effective pruning with bounds, as shown in our BP-Cubing algorithms, discussed in Section 7. In later discussions, we use the term "data cube" to refer to a standard or subdata cube.

Definition 3 (Subdata cube). Consider dimensions $A_{1}, \ldots, A_{n}$ and $B_{1}, \ldots, B_{k}$ and values

$$
b_{1} \in \operatorname{domain}\left(B_{1}\right), \ldots, b_{k} \in \operatorname{domain}\left(B_{k}\right) .
$$

The groups aggregating tuples that satisfy $B_{i}=b_{i}$ for $1 \leq$ $i \leq k$ form a subdata cube, or simply a subcube, denoted as Cube $\left(A_{1}, \ldots, A_{n}\right) \mid b_{1}, \ldots, b_{k}$. The core of the subdata cube comprises MSPs with $B_{i}=b_{i}$ for $1 \leq i \leq k$. $B_{1}, \ldots, B_{k}$ are conditional dimensions for the subcube.

Example 2. Continuing with Example 1, consider

$$
\text { Cube(Product, SalesMan, City)|Mar. }
$$

There are three MSPs for the data cube: (Mar, TV, Peter, Perth), (Mar, TV, John, Perth), and 
(Mar, TV, John, Sydney). Tighter bounds can be obtained on subcubes. Indeed, the tightest upper bound for Sum(Sale) for the data cube is $100+100+100=300$, and the tightest lower bound is $\operatorname{Min}(\{100,100,100\})=100$.

Based on Observation 1, we can see that a data cube can be bounded from its MSPs. However, exhaustively checking the power set of MSPs is equivalent to computing the complete data cube and is not computationally feasible. We define boundable aggregate functions as follows.

Definition 4 (Boundable aggregate function). An aggregate function $F$ is boundable for a data cube if some upper and lower bounds of $F$ for the data cube can be determined by some algorithm with a single scan of some auxiliary aggregate values of the MSPs of the data cube. We will use $\overline{F(\operatorname{Cube}(X))}$ and $F(\mathrm{Cube}(X))$ to denote, respectively, the upper and lower bounds, computed by a given single-scan algorithm. ${ }^{7}$

It should be pointed out that the bounds computed may not be the tightest. To ensure the effectiveness of pruning, we aim to derive bounds that are as tight as possible. Let us use some example aggregate functions to explain this definition.

Example 3. Consider the aggregate function Count and measure $X$ with $n$ MSPs $X_{1}, \ldots, X_{n}$, where

$$
\operatorname{Count}(X)=\operatorname{Sum}\left(\left\{\operatorname{Count}\left(X_{i}\right) \mid i=1 \ldots n\right\}\right) .
$$

The auxiliary aggregate function is Count. For any partition $g$ of Cube $(X)$, the number of tuples in $g$ is not larger than the total number of tuples of all MSPs; in other words, $\operatorname{Count}(g) \leq \operatorname{Sum}\left(\left\{\operatorname{Count}\left(X_{i}\right) \mid i=1 \ldots n\right\}\right)$. On the other hand, we also have

$$
\operatorname{Count}(g) \geq \operatorname{Min}\left(\left\{\operatorname{Count}\left(X_{i}\right) \mid i=1 \ldots n\right\}\right) .
$$

As a result, $\operatorname{Sum}\left(\left\{\operatorname{Count}\left(X_{i}\right) \mid i=1 \ldots n\right\}\right)$ is an upper bound, and $\operatorname{Min}\left(\left\{\operatorname{Count}\left(X_{i}\right) \mid i=1 \ldots n\right\}\right)$ is a lower bound. They can be obtained by a single scan of the auxiliary aggregates of MSPs. Therefore, Count $(\mathrm{Cube}(X))$ is boundable.

We now give an example of aggregate functions whose bounds can be infinite.

Example 4. Given measure $X$ and aggregate function $1 /$ Sum, consider $\operatorname{Cube}(X)$ with MSPs $X_{1}, \ldots, X_{n}$. Then, $1 / \operatorname{Sum}(X)=1 / \operatorname{Sum}\left(\left\{\operatorname{Sum}\left(X_{i}\right) \mid i=1 \ldots n\right\}\right)$. As will be explained later in Tables 3 and 4 , the signs of $\operatorname{Sum}\left(X_{i}\right)$ are important for computing the bounds:

1. If $\operatorname{Sum}\left(X_{i}\right)>0$ for all $i=1 \ldots n$, then the upper and lower bounds are computed from, respectively, the positive lower and upper bounds of $\operatorname{Sum}(\operatorname{Cube}(X))$ :

$$
\overline{1 / \operatorname{Sum}(\operatorname{Cube}(X))}=1 / \operatorname{Min}\left(\left\{\operatorname{Sum}\left(X_{i}\right) \mid i=1 \ldots n\right\}\right)
$$

7. We will write $\overline{F(\operatorname{Cube}(X))}$ and $F(\operatorname{Cube}(X))$ to mean, respectively, the upper and lower bounds computed by our algorithm. That algorithm is the sum of the bounding formulas presented in this paper.

\begin{tabular}{|c|c|}
\hline$F$ & $\overline{F(\operatorname{Cube}(X))} ; F(\operatorname{Cube}(X))$ \\
\hline Count & $\operatorname{Sum}_{i} \operatorname{Count}\left(X_{i}\right) ; \operatorname{Min}_{i} \operatorname{Count}\left(X_{i}\right)$ \\
\hline Max & $\operatorname{Max}_{i} \operatorname{Max}\left(X_{i}\right) ; \operatorname{Min}_{i} \operatorname{Max}\left(X_{i}\right)$ \\
\hline Min & $\operatorname{Max}_{i} \operatorname{Min}\left(X_{i}\right) ; \operatorname{Min}_{i} \operatorname{Min}\left(X_{i}\right)$ \\
\hline Sum & $\begin{array}{c}\underset{\operatorname{Sum}}{\operatorname{Sum}\left(X_{i}\right)>0} \operatorname{Sum}\left(X_{i}\right) \text { if (a) } \\
\underset{i}{\operatorname{Max} \operatorname{Sum}\left(X_{i}\right) \text { otherwise }} ; \quad \operatorname{Sum}\left(X_{i}\right)<0 \\
\operatorname{Min} \operatorname{Sum}\left(X_{i}\right) \text { if (b) } \\
\left.X_{i}\right) \text { otherwise }\end{array}$ \\
\hline
\end{tabular}

TABLE 2

The Bounds of Base Aggregate Functions

Given a data set, $X$ is the measure, and $X_{1}, \ldots, X_{n}$ are the MSPs. (a) There is $i$ such that $\operatorname{Sum}\left(X_{i}\right)>0$. (b) There is $i$ such that $\operatorname{Sum}\left(X_{i}\right)<0$.

and

$$
\underline{1 / \operatorname{Sum}(\operatorname{Cube}(X))}=1 / \operatorname{Sum}\left(\left\{\operatorname{Sum}\left(X_{i}\right) \mid i=1 \ldots n\right\}\right) .
$$

2. If $\operatorname{Sum}\left(X_{i}\right)<0$ for all $i=1 \ldots n$, then the upper and lower bounds are computed from, respectively, the negative lower and upper bounds of $\operatorname{Sum}(\operatorname{Cube}(X))$ as

$\overline{1 / \operatorname{Sum}(\operatorname{Cube}(X))}=1 / \operatorname{Sum}\left(\left\{\operatorname{Sum}\left(X_{i}\right) \mid i=1 \ldots n\right\}\right)$

and

$1 / \operatorname{Sum}(\operatorname{Cube}(X))=1 / \operatorname{Max}\left(\left\{\operatorname{Sum}\left(X_{i}\right) \mid i=1 \ldots n\right\}\right)$.

3. For general cases, where $\operatorname{Sum}\left(X_{i}\right)$ can be positive or negative, the upper bound $\overline{1 / \operatorname{Sum}(\operatorname{Cube}(X))}$ is computed from the smallest $\operatorname{Sum}(x) \geq 0$ for any partition in the cube. For any group $g$ of $\operatorname{Cube}(X)$, $\operatorname{Sum}(g)$ can be the sum of any positive or negative MSP aggregates and can have a minimum of 0 . Thus, the upper bound for $1 / \operatorname{Sum}(\operatorname{Cube}(X))$ is $\infty$. Similarly, the lower bound $1 / \operatorname{Sum}(\operatorname{Cube}(X))$ is computed from the largest $\operatorname{Sum}(x)<0$ for any partition in the cube, which can be a negative approaching 0 . Therefore, the lower bound for $1 / \operatorname{Sum}(\operatorname{Cube}(X))$ is $-\infty$.

Computing the upper and lower bounds for arithmetic expressions of base aggregate functions such as 1 /Sum is summarized later in Table 4 .

\section{Bounding Base Aggregate Functions}

We now consider how we can bound the base aggregate functions of SQL.

Theorem 1. The base aggregate functions Count, Min, Max, and Sum are boundable, and their bounds are listed in Table 2.

We use the following example for Sum to explain the reasoning for the bounds of Table 2 and how they are obtained by a single scan of MSPs.

Example 5. Suppose we are to compute a data cube on measure $X$, which may take positive or negative values. Let the MSPs of $X$ be $X_{1}, \ldots, X_{n}$. With a single scan of $X_{1}, \ldots, X_{n}$, we have $\operatorname{Sum}\left(X_{i}\right)(1 \leq i \leq n)$ and the positive/negative and maximal/minimal values among them: 
TABLE 3

The Sign Bounds of Base Aggregate Functions

\begin{tabular}{|c|c|c|}
\hline$F$ & $\overline{F^{+}(\operatorname{Cube}(X))} ; F^{+}(\operatorname{Cube}(X))$ & $\overline{F^{-}(\operatorname{Cube}(X))} ; F^{-}(\operatorname{Cube}(X))$ \\
\hline Count & $\operatorname{Sum}_{i} \operatorname{Count}\left(X_{i}\right) ; \operatorname{Min}_{i} \operatorname{Count}\left(X_{i}\right)$ & $\mathrm{n} / \mathrm{a} ; \mathrm{n} / \mathrm{a}$ \\
\hline $\operatorname{Max}$ & $\begin{array}{c}\operatorname{Max}_{\operatorname{Max}\left(X_{i}\right) \geq 0} \operatorname{Max}\left(X_{i}\right) \\
\underset{\operatorname{Min}}{\operatorname{Min}\left(X_{i}\right) \geq 0} \\
\operatorname{Max}\left(X_{i}\right)\end{array}$ & $\begin{array}{l}\operatorname{Max}_{\operatorname{Max}\left(X_{i}\right)<0} \operatorname{Max}\left(X_{i}\right) ; \\
\operatorname{Min}_{\operatorname{Max}\left(X_{i}\right)<0} \operatorname{Max}\left(X_{i}\right)\end{array}$ \\
\hline Min & $\begin{array}{c}\operatorname{Max} \operatorname{Min}\left(X_{i}\right) \\
\operatorname{Min}\left(X_{i}\right) \geq 0 \\
\operatorname{Min}_{\operatorname{Min}\left(X_{i}\right) \geq 0} \operatorname{Min}(X)\end{array}$ & $\begin{array}{l}\underset{\operatorname{Max}}{\operatorname{Min}\left(X_{i}\right)<0} \operatorname{Min}\left(X_{i}\right) \\
\operatorname{Min}_{\operatorname{Min}\left(X_{i}\right)<0} \operatorname{Min}\left(X_{i}\right)\end{array}$ \\
\hline Sum & $\begin{array}{l}\underset{\operatorname{Sum}}{\operatorname{Sum}\left(X_{i}\right) \geq 0} \operatorname{Sum}\left(X_{i}\right) ; \\
\text { if } \operatorname{Sum}\left(X_{i}\right) \geq 0 \text { for } i=1 . . n \\
\underset{i}{\operatorname{Min} \operatorname{Sum}\left(X_{i}\right), \text { otherwise } 0 .}\end{array}$ & $\begin{array}{l}\text { If } \operatorname{Sum}\left(X_{i}\right)<0 \text { for } i=1 . . n \\
\operatorname{Max} \operatorname{Sum}\left(X_{i}\right) \text {, otherwise 0; } \\
\underset{i}{\operatorname{Sum}} \operatorname{Sum}\left(X_{i}\right)<0\end{array}$ \\
\hline
\end{tabular}

$X$ is the measure of a data set, and the MSPs for the data cube are $X_{1}, \ldots, X_{n}$.

1. If there exists $i$ such that $\operatorname{Sum}\left(X_{i}\right)>0$, then the sum for any group in $\operatorname{Cube}(X)$ is not greater than the sum of all such positive $\operatorname{Sum}\left(X_{i}\right)$. Otherwise, $\operatorname{Sum}\left(X_{i}\right) \leq 0$ for $i=1 \ldots n$; the sum for any group is not greater than the maximal $\operatorname{Sum}\left(X_{i}\right)$. Thus,

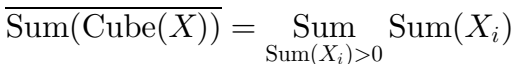

if there exists $\operatorname{Sum}\left(X_{i}\right)>0$;

$$
\overline{\operatorname{Sum}(\operatorname{Cube}(X))}=\underset{i}{\operatorname{Max} \operatorname{Sum}}\left(X_{i}\right) \text {, otherwise. }
$$

2. If there exists $i$ such that $\operatorname{Sum}\left(X_{i}\right)<0$, then the sum of any group in $C u b e(X))$ is not less than the sum of all such $\operatorname{Sum}\left(X_{i}\right)$. Otherwise, $\operatorname{Sum}\left(X_{i}\right) \geq 0$ for all $i=1 \ldots n$; the sum of any group is not less than the minimal $\operatorname{Sum}\left(X_{i}\right)$. Therefore,

$$
\underline{\operatorname{Sum}(\operatorname{Cube}(X))}=\underset{\operatorname{Sum}\left(X_{i}\right)<0}{\operatorname{Sum}\left(X_{i}\right)}
$$

if there exists $\operatorname{Sum}\left(X_{i}\right)<0$;

$$
\underline{\operatorname{Sum}(\operatorname{Cube}(X))}=\underset{i}{\operatorname{MinSum}}\left(X_{i}\right), \text { otherwise. }
$$

To bound functions that are arithmetic expressions of base aggregate functions, sometimes, the sign bounds of aggregations need to be computed (see Section 5). These include positive upper bound $\left(\overline{F^{+}}\right)$, positive lower bound $\left(\underline{F^{+}}\right)$, negative upper bound $\left(\overline{F^{-}}\right)$, and negative lower bound $\left(\underline{F^{-}}\right)$, where $F$ is either a base aggregate function Min, Max, Sum, or Count or an arithmetic expression of base aggregate functions. $\overline{F^{+} \text {Cube }(X)}$ is defined to be a real $l \geq 0$ obtained by a single-scan algorithm such that $l \geq F(g) \geq 0$ for any partition $g$ in $\operatorname{Cube}(X)$. The other sign bounds can be defined similarly. The positive (negative) bounds are applicable only if there are nonnegative (negative) aggregates among the MSPs. The sign bounds of base aggregate functions are listed in Table 3.

We explain how the sign bounds for Sum are computed. Computation for other functions is straightforward. We discuss how we can compute the positive bounds of Sum,

\begin{tabular}{|c|c|}
\hline op & bounds for the expression $\left(E_{1}\right.$ op $\left.E_{2}\right)$ \\
\hline+ & $\overline{\left(E_{1}+E_{2}\right)}=\overline{E_{1}}+\overline{E_{2}},\left(\underline{\left.E_{1}+E_{2}\right)}=\underline{E_{1}}+\underline{E_{2}}\right.$ \\
\hline- & $\overline{\left(E_{1}-E_{2}\right)}=\overline{E_{1}}-\underline{E_{2}}, \overline{\left(E_{1}-E_{2}\right)}=\bar{E}_{1}-\overline{\overline{E_{2}}}$ \\
\hline$\times$ & $\overline{\left(E_{1} \times E_{2}\right)}=\operatorname{Max}\left(\overline{E_{1}^{+}} \times \overline{\overline{E_{2}^{+}}}, \underline{\underline{E_{1}^{-}} \times E_{2}^{-}}, \overline{E_{1}^{+}} \times \overline{E_{2}^{-}}, \overline{E_{1}^{-}} \times \underline{E_{2}^{+}}\right)$ \\
\hline & $\underline{\left(E_{1} \times E_{2}\right)}=\operatorname{Min}\left(\underline{E_{1}^{+}} \times E_{2}^{+}, \overline{E_{1}^{-}} \times \overline{E_{2}^{-}}, \overline{E_{1}^{+}} \times \overline{E_{2}^{-}}, \frac{E_{1}^{-}}{\bar{F}^{+}} \times \overline{E_{2}^{+}}\right)$ \\
\hline 1 & $\begin{array}{l}\left(E_{1} / E_{2}\right)=\operatorname{Max}\left(E_{1}^{+} / E_{2}^{+}, E_{1}^{-} / E_{2}^{-}, E_{1}^{+} / E_{2}^{-}, E_{1}^{-} / E_{2}^{+}\right) \\
\left(E_{1} / E_{2}\right)=\operatorname{Min}\left(E_{1}^{+} / \overline{E_{2}^{+}}, \underline{\underline{E_{1}^{-}} / E_{2}^{-},}, \underline{\overline{E_{1}^{+}}} / \overline{E_{2}^{-}}, E_{1}^{-} / E_{2}^{+}\right)\end{array}$ \\
\hline
\end{tabular}
with discussions on the negative bounds mirror the same
TABLE 4

Bounding Arithmetic Expressions

The short notations $E_{1}, E_{2}$, and $\left(E_{1}\right.$ op $\left.E_{2}\right)$ denote $E_{1}(\operatorname{Cube}(X))$, $E_{2}(\operatorname{Cube}(X))$, and $\left(E_{1}\right.$ op $\left.E_{2}\right)(\operatorname{Cube}(X))$, respectively, where $X$ is the measure of a data set. When the operator is " $X$ " or "," to bound $\left(E_{1}\right.$ op $\left.E_{2}\right)$, not all four kinds of computation are applicable. As indicated, the signs of $E_{1}\left(X_{i}\right)$ and $E_{2}\left(X_{i}\right)(i=1 \ldots n)$ decide the applicable computation.

case as in positive bounds. The positive upper bound is the sum of all positive sums of the MSPs. To compute the positive lower bound, two cases exist: 1) if $\operatorname{Sum}\left(X_{i}\right) \geq 0$ for all $X_{i}(1 \leq i \leq n)$, then the positive lower bound is the minimal $\operatorname{Sum}\left(X_{i}\right) \geq 0$ and 2) if the sums of MSPs include both negative and positive ones, then the sum of any set of MSPs can be as low as 0 , and so, 0 is the positive lower bound.

\section{Bounding Arithmetic EXPRessions of BASE AgGREgATE FunCtIONS}

Consider a data cube $\operatorname{Cube}(X)$ and an arithmetic expression of auxiliary aggregates $E=E_{1}$ op $E_{2}$, where $E_{1}$ and $E_{2}$ are base aggregate functions or arithmetic expressions of base aggregates. If the operator is " + " or ",- " then the upper (lower) bounds of $E(\operatorname{Cube}(X))$ can be computed from the upper (lower) bounds of $E_{1}(\operatorname{Cube}(X))$ and $E_{2}(\operatorname{Cube}(X))$. If the operator is " $X$ " or " $/$, , then we need to use the sign bounds of $E_{1}(\operatorname{Cube}(X))$ and $E_{2}(\operatorname{Cube}(X))$ to bound $E(\operatorname{Cube}(X))$.

Proposition 1. Given $\operatorname{Cube}(X)$ on measure $X$ and an arithmetic expression $E=E_{1}$ op $E_{2}$, where the operator is " + ," "-," " $\times$, , or "I/," and $E_{1}$ and $E_{2}$ are base aggregate functions or arithmetic expressions of base aggregates, $\overline{E(\mathrm{Cube}(X))}$ and $E(\operatorname{Cube}(X))$ can be computed from the bounds or sign bounds of $E_{1}(\operatorname{Cube}(X))$ and $E_{2}(\operatorname{Cube}(X))$, as shown in Table 4.

Proof. Let $X_{1}, \ldots, X_{n}$ be the MSPs of $\operatorname{Cube}(X)$. We consider each of the four possible operators:

1. $E_{1}+E_{2}$. By the definition of upper bound, for any group $g \in \operatorname{Cube}(X), \quad E_{1}(g) \leq \overline{E_{1}(\operatorname{Cube}(X))}$, and $E_{2}(g) \leq \overline{E_{2}(\operatorname{Cube}(X))}$. We have

$$
E_{1}(g)+E_{2}(g) \leq \overline{E_{1}(\operatorname{Cube}(X))}+\overline{E_{2}(\operatorname{Cube}(X))} .
$$

Thus,

$$
\overline{\left(E_{1}+E_{2}\right)(\operatorname{Cube}(X))}=\overline{E_{1}(\operatorname{Cube}(X))}+\overline{E_{2}(\operatorname{Cube}(X))} .
$$

The proof is similar for

$$
\underline{\left(E_{1}+E_{2}\right)(\operatorname{Cube}(X))}=\underline{E_{1}(\operatorname{Cube}(X))}+\underline{E_{2}(\operatorname{Cube}(X))} .
$$


2. $E_{1}-E_{2}$. Given $g \in \operatorname{Cube}(X), E_{1}(g) \leq \overline{E_{1}(\operatorname{Cube}(X))}$ and $E_{2}(g) \geq E_{2}(\operatorname{Cube}(X))$. Thus,

$$
E_{1}(g)-E_{2}(g) \leq \overline{E_{1}(\operatorname{Cube}(X))}-\underline{E_{2}(\operatorname{Cube}(X))}
$$

and

$\overline{\left(E_{1}-E_{2}\right)(\operatorname{Cube}(X))}=\overline{E_{1}(\operatorname{Cube}(X))}-\underline{E_{2}(\operatorname{Cube}(X))}$.

The proof is similar for

$$
\underline{\left(E_{1}-E_{2}\right)(\operatorname{Cube}(X))}=\underline{E_{1}(\operatorname{Cube}(X))}-\overline{E_{2}(\operatorname{Cube}(X))} .
$$

3. $E_{1} \times E_{2}$. Let $g$ be a group of $\operatorname{Cube}(X)$. Let $Y_{1}, Y_{2}, \ldots, Y_{k}$ be the MSPs that are contained in $g$. Based on their signs under $E_{1}$, we form two subsets from these MSPs:

$$
\begin{aligned}
& P E_{1}=\left\{Y_{j} \mid E_{1}\left(Y_{j}\right) \geq 0,1 \leq j \leq k\right\}, \\
& N E_{1}=\left\{Y_{j} \mid E_{1}\left(Y_{j}\right)<0,1 \leq j \leq k\right\} .
\end{aligned}
$$

Similarly, based on their signs under $E_{2}$, we form the following subsets from these MSPs:

$$
\begin{aligned}
& P E_{2}=\left\{Y_{j} \mid E_{2}\left(Y_{j}\right) \geq 0,1 \leq j \leq k\right\}, \text { and } \\
& N E_{2}=\left\{Y_{j} \mid E_{2}\left(Y_{j}\right)<0,1 \leq j \leq k\right\} .
\end{aligned}
$$

For the upper bound, we first consider the case where $P E_{1} \neq \emptyset$ and $P E_{2} \neq \emptyset$ or $N E_{1} \neq \emptyset$ and $N E_{2} \neq \emptyset . E_{1}(g) \times E_{2}(g)$ can have positive values. Thus,

$$
\begin{array}{r}
E_{1}(g) \times E_{2}(g) \leq \operatorname{Max}\left(\overline{E_{1}^{+}(\operatorname{Cube}(X))} \times \overline{E_{2}^{+}(\operatorname{Cube}(X))},\right. \\
\underline{E_{1}^{-}(\operatorname{Cube}(X))} \times \underline{\left.E_{2}^{-}(\operatorname{Cube}(X))\right)} .
\end{array}
$$

Otherwise, $E_{1}(g) \times E_{2}(g)$ can only be negative. We have

$$
\begin{aligned}
& E_{1}(g) \times E_{2}(g) \leq \operatorname{Max}\left(\underline{E_{1}^{+}(\operatorname{Cube}(X))} \times \overline{E_{2}^{-}(\operatorname{Cube}(X))},\right. \\
& \overline{E_{1}^{-}(\operatorname{Cube}(X))} \times \underline{\left.E_{2}^{+}(\operatorname{Cube}(X))\right)} .
\end{aligned}
$$

For all cases, by combining the two inequalities with $E_{1}(g) \times E_{2}(g)$ on the left-hand side, we get $\overline{\left(E_{1} \times E_{2}\right)(\operatorname{Cube}(X))}$ :

$$
\begin{gathered}
\operatorname{Max}\left(\overline{E_{1}^{+}(\operatorname{Cube}(X))} \times \overline{E_{2}^{+}(\operatorname{Cube}(X)),}\right. \\
\frac{E_{1}^{-}(\operatorname{Cube}(X))}{\overline{E_{1}^{+}(\operatorname{Cube}(X))}} \times \overline{E_{2}^{-}(\operatorname{Cube}(X)),} \\
\overline{\overline{E_{1}^{-}(\operatorname{Cube}(X))},} \\
\times \underline{\left.E_{2}^{+}(\operatorname{Cube}(X))\right)} .
\end{gathered}
$$

The proof for the lower bound is quite similar to that for the upper bound. The possible positive values of $g$ are

$$
\geq \frac{\operatorname{Min}\left(\underline{E_{1}^{+}(\operatorname{Cube}(X))} \times \underline{E_{2}^{+}(\operatorname{Cube}(X))},\right.}{\left.\overline{E_{1}^{-}(\operatorname{Cube}(X))} \times \overline{E_{2}^{-}(\operatorname{Cube}(X))}\right)}
$$

and the possible negative values of $g$ are

$$
\begin{aligned}
\geq & \operatorname{Min}\left(\overline{E_{1}^{+}(\operatorname{Cube}(X))} \times \underline{E_{2}^{-}(\operatorname{Cube}(X))},\right. \\
& \left.\underline{E_{1}^{-}(\operatorname{Cube}(X))} \times \overline{E_{2}^{+}(\operatorname{Cube}(X))}\right) .
\end{aligned}
$$

By combining the last two inequalities, we get $\underline{\left(E_{1} \times E_{2}\right)(\operatorname{Cube}(X))}$ :

$$
\begin{gathered}
\operatorname{Min}\left(\frac{\left(E_{1}^{+}(\operatorname{Cube}(X))\right.}{\overline{E_{1}^{-}(\operatorname{Cube}(X))}} \times \overline{\underline{E_{2}^{+}(\operatorname{Cube}(X))}} \times \overline{E_{2}^{-}(\operatorname{Cube}(X))},\right. \\
\overline{E_{1}^{+}(\operatorname{Cube}(X))} \times \overline{E_{2}^{-}(\operatorname{Cube}(X))}, \\
\underline{E_{1}^{-}(\operatorname{Cube}(X))} \times \overline{\left.E_{2}^{+}(\operatorname{Cube}(X))\right)} .
\end{gathered}
$$

4. $E_{1} / E_{2}$. The proof is similar to that for the operator " $x$ " and is omitted.

Example 6. Consider a data cube $\operatorname{Cube}(X)$, where the measure $X$ can be positive or negative, and the aggregate function Avg. Suppose the MSPs are $X_{1}, \ldots, X_{n}$. For any $\operatorname{MSP} X_{i}$, we note that $\operatorname{Avg}\left(X_{i}\right)=\operatorname{Sum}\left(X_{i}\right) / \operatorname{Count}\left(X_{i}\right)$, and Count $\left(X_{i}\right)$ is always positive. Following Table 4, $\overline{\operatorname{Avg}(\operatorname{Cube}(X))}$ is

$$
\begin{aligned}
& \operatorname{Max}\left(\overline{\operatorname{Sum}^{+}(\operatorname{Cube}(X))} / \underline{\text { Count }^{+}(\operatorname{Cube}(X))},\right. \\
& \left.\overline{\operatorname{Sum}^{-}(\operatorname{Cube}(X))} / \overline{\operatorname{Count}^{+}(\operatorname{Cube}(X))}\right)
\end{aligned}
$$

and $\operatorname{Avg}(\operatorname{Cube}(X))$ is

$$
\begin{array}{r}
\operatorname{Min}\left(\frac{\operatorname{Sum}^{+}(\operatorname{Cube}(X))}{\operatorname{Count}^{+}(\operatorname{Cube}(X))},\right. \\
\left.\underline{\operatorname{Sum}^{-}(\operatorname{Cube}(X))} / \operatorname{Count}^{+}(\operatorname{Cube}(X))\right) .
\end{array}
$$

If there exists some $i$ such that $\operatorname{Sum}\left(X_{i}\right)>0$, then

$\overline{\operatorname{Avg}(\operatorname{Cube}(X))}=\overline{\operatorname{Sum}^{+}(\operatorname{Cube}(X))} / \operatorname{Count}(\operatorname{Cube}(X))$, and

$\operatorname{Avg}(\operatorname{Cube}(X))=\operatorname{Sum}^{+}(\operatorname{Cube}(X)) / \overline{\operatorname{Count}(\operatorname{Cube}(X))}$.

Otherwise, $\operatorname{Sum}\left(X_{i}\right) \leq 0$ for all $i=1 \ldots n$; then, we have

$\overline{\operatorname{Avg}(\operatorname{Cube}(X))}=\overline{\operatorname{Sum}^{-}(\operatorname{Cube}(X))} / \overline{\operatorname{Count}(\operatorname{Cube}(X))}$, and

$\operatorname{Avg}(\operatorname{Cube}(X))=\operatorname{Sum}^{-}(\operatorname{Cube}(X)) / \operatorname{Count}(\operatorname{Cube}(X))$.

We then follow Table 3 to compute the sign bounds in the above expressions.

The sign bounds for base aggregate functions are listed in Table 3. We now discuss how we can derive the sign bounds for arithmetic expressions. Note that in Table 5, positive (negative) bounds are applicable only if there exists an MSP $X_{i}$ in the cube such that

$$
\left(E_{1}\left(X_{i}\right) \text { op } E_{2}\left(X_{i}\right)\right) \geq 0\left(\left(E_{1}\left(X_{i}\right) \text { op } E_{2}\left(X_{i}\right)\right)<0\right) .
$$


TABLE 5

The Sign Bounds of Arithmetic Expressions

\begin{tabular}{|c|c|c|}
\hline op & 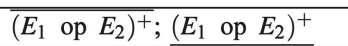 & $\overline{\left(E_{1} \text { op } E_{2}\right)^{-}} ;\left(E_{1} \text { op } E_{2}\right)^{-}$ \\
\hline+ & $\begin{array}{l}\overline{E_{1}}+\overline{E_{2}} ; \\
\text { if } \underline{E_{1}}+\underline{E_{2}} \geq 0, \underline{E_{1}}+\underline{E_{2}} \text {, other- } \\
\text { wise } 0 .\end{array}$ & $\begin{array}{l}\text { if } \overline{E_{1}}+\overline{E_{2}}<0, \overline{E_{1}}+\overline{E_{2}} \text {, other- } \\
\text { wise } 0 ; \\
E_{1}+\underline{E_{2}}\end{array}$ \\
\hline- & $\begin{array}{l}\overline{E_{1}}-E_{2} \\
\text { if } \underline{E_{1}}-\overline{E_{2}} \geq 0, \underline{E_{1}}-\overline{E_{2}} \text {, other- } \\
\text { wise } 0 \text {. }\end{array}$ & $\begin{array}{l}\text { if } \overline{E_{1}}-E_{2}<0, \overline{E_{1}}-\underline{E_{2}} \text {, other- } \\
\text { wise } 0 ; \\
E_{1}-\overline{E_{2}}\end{array}$ \\
\hline$x$ & $\begin{array}{l}\operatorname{Max}\left(\overline{E_{1}^{+}} \times \overline{E_{2}^{+}}, \overline{E_{1}^{-}} \times \overline{E_{2}^{-}}\right) ; \\
\operatorname{Min}\left(E_{1}^{+} \times E_{2}^{+}, \overline{\overline{E_{1}^{-}}} \times \overline{\overline{E_{2}^{-}}}\right)\end{array}$ & $\begin{array}{l}\operatorname{Max}\left(\overline{E_{1}^{+}} \times \overline{E_{2}^{-}}, \overline{E_{1}^{-}} \times \overline{E_{2}^{+}}\right) ; \\
\operatorname{Min}\left(\overline{\overline{E_{1}^{+}}} \times \overline{E_{2}^{-}},{\overline{E_{1}^{-}}}^{-} \times \overline{E_{2}^{+}}\right)\end{array}$ \\
\hline / & $\begin{array}{l}\operatorname{Max}\left(\overline{\overline{E_{1}^{+}}} / \overline{E_{2}^{+}}, E_{1}^{-} / \overline{E_{2}^{-}}\right) ; \\
\operatorname{Min}\left(E_{1}^{+} / \overline{\overline{E_{2}^{+}}}, \overline{E_{1}^{-}} / E_{2}^{-}\right)\end{array}$ & $\begin{array}{l}\operatorname{Max}\left(\left(_{1}^{+} / \overline{E_{2}^{-}}, \overline{E_{1}^{-}} / \overline{E_{2}^{+}}\right) ;\right. \\
\operatorname{Min}\left(\overline{\overline{E_{1}^{+}}} / \overline{\overline{E_{2}^{-}}}, E_{1}^{-} / E_{2}^{+}\right)\end{array}$ \\
\hline
\end{tabular}

Short notations $E_{1}, \quad E_{2}$, and $\left(E_{1}\right.$ op $\left.E_{2}\right)$ denote $E_{1}(\operatorname{Cube}(X))$, $E_{2}(\operatorname{Cube}(X))$, and $\left(E_{1}\right.$ op $\left.E_{2}\right)(\operatorname{Cube}(X))$, respectively, where $X$ is the measure of a data set.

For example, the positive upper bound

$$
\overline{\left(E_{1}+E_{2}\right)(\operatorname{Cube}(X))^{+}}
$$

is applicable only if there exists $X_{i}$ such that

$$
E 1\left(X_{i}\right)+E_{2}\left(X_{i}\right) \geq 0 .
$$

For the operators "+" and "-, when $E_{1}+E_{2}$ or $E_{1}-E_{2}$ can be positive or negative for MSPs, the positive lower and negative upper bounds for both expressions are 0 : this is because the combination of positive and negative MSPs can produce the aggregate value of 0 . For the operators " $x$ " and "/," the signs of $E_{1}$ and $E_{2}$ for MSPs decide the applicable computation. We use an example to explain Table 5.

Example 7. Given data cube $\operatorname{Cube}(X)$ with MSPs $X_{1}, \ldots, X_{n}$, consider the aggregate function

$$
E(X)=1 /(\operatorname{Max}(X)+\operatorname{Min}(X)) .
$$

To compute $\overline{E(\operatorname{Cube}(X))}$, three cases can arise:

Case 1. $\operatorname{Min}\left(X_{i}\right) \geq 0$ for all $i=1 \ldots n$. It follows that $\operatorname{Max}\left(X_{i}\right) \geq 0$ for all $i=1 \ldots n$. Then,

$$
\overline{E(\operatorname{Cube}(X))}=1 /\left(\underline{\operatorname{Max}^{+}(\operatorname{Cube}(X))}+\underline{\operatorname{Min}^{+}(\operatorname{Cube}(X))}\right) .
$$
Then,

Case 2. $\operatorname{Max}\left(X_{i}\right) \leq 0$ and $\operatorname{Min}\left(X_{i}\right) \leq 0$ for all $i=1 \ldots n$.

$\overline{E(\operatorname{Cube}(X))}=1 /\left(\overline{\operatorname{Max}^{-}(\operatorname{Cube}(X))}+\overline{\operatorname{Min}^{-}(\operatorname{Cube}(X))}\right)$.

Case 3. $\operatorname{Max}\left(X_{i}\right)$ and $\operatorname{Min}\left(X_{i}\right)$ can be positive or negative $(1 \leq i \leq n)$. Based on Table 4 , we have $\overline{E(\operatorname{Cube}(X))}=1 /(\operatorname{Max}()+\operatorname{Min}())^{+}(\operatorname{Cube}(X))$. Based on Table 5, $(\operatorname{Max}()+\operatorname{Min}())^{+}(C u b e(X))$ is 0 , which means that the upper bound for $1 /(\operatorname{Max}()+\operatorname{Min}())(\operatorname{Cube}(X))$ is $\infty$.

Proposition 1 leads to the following theorem about bounding arithmetic expressions of base aggregate functions.

Theorem 2. Given a data cube on measure $X$, an arithmetic expression $E=E_{1}$ op $E_{2}$ of base aggregate functions, where the operator is,,$+- \times$, or $/ . E$ is boundable for $\operatorname{Cube}(X)$, and its bounds can be computed following Tables 2, 3, 4, and 5.
Proof. By Proposition 1, we can compute the bounds for an arithmetic expression from the bounds or sign bounds of the operand expressions, as listed in Table 4. Table 5 shows how the sign bounds of any expression can be computed from the bounds for base aggregate functions. Tables 2 and 3 demonstrate that a single scan of the MSPs of a data cube can compute the bounds of all base aggregate functions. As a result, all arithmetic expressions of base aggregate functions are boundable, and the bounds can be computed following Tables 2, 3, 4, and 5 .

We now show how we can compute the upper bound for the complex aggregate function Var based on the theorem.

Example 8. The Var for $X=\left\{x_{i} \mid i=1 \ldots N\right\}$ is defined as ${ }^{8}$ $\operatorname{Var}(X)=\frac{\sum_{i}\left(x_{i}-\bar{X}\right)^{2}}{N}$, where $\bar{X}$ denotes the Avg of $X$. It can be rewritten as follows:

$$
\begin{aligned}
\operatorname{Var}(X) & =\frac{\sum_{i}\left(x_{i}^{2}-2 \times \bar{X} \times x_{i}+\bar{X}^{2}\right)}{N}, \\
& =\frac{\sum_{i} x_{i}^{2}}{N}-\frac{2 \times \bar{X} \times \sum_{i} x_{i}}{N}+\frac{N \times \bar{X}^{2}}{N}, \\
& =\frac{\sum_{i} x_{i}^{2}}{N}-2 \times \bar{X}^{2}+\bar{X}^{2}=\frac{\sum_{i} x_{i}^{2}}{N}-\bar{X}^{2}, \\
& =\frac{\operatorname{Sum} x_{i}^{2}}{\operatorname{Count}(X)}-\left(\frac{\operatorname{Sum}(X)}{\operatorname{Count}(X)}\right)^{2} .
\end{aligned}
$$

Let $\operatorname{QSum}(X)$ denote $\operatorname{Sum}_{i} x_{i}^{2}$. It follows that $\operatorname{Var}(X)$ is an arithmetic expression of $\mathrm{QSum}(X), \operatorname{Sum}(X)$, and $\operatorname{Count}(X)$. Consider a data cube $\operatorname{Cube}(X)$ with MSPs $X_{1}, \ldots, X_{n}$. Based on Table 4 , we have

$$
\begin{aligned}
\overline{\operatorname{Var}(\operatorname{Cube}(X))}= & \overline{\left(\frac{\mathrm{QSum}()}{\operatorname{Count}()}\right)(\operatorname{Cube}(X))} \\
& -\left(\frac{\operatorname{Sum}()}{\operatorname{Count}()}\right)^{2}(\operatorname{Cube}(X)) .
\end{aligned}
$$

We next consider each of the two subexpressions separately.

We first consider $\overline{\left(\frac{\mathrm{QSum}()}{\operatorname{Count}()}\right)(\operatorname{Cube}(X))}$ : For any MSP $X_{i}$, $\operatorname{QSum}\left(X_{i}\right)$ and $\operatorname{Count}\left(X_{i}\right)$ are always positive. By following Table 4, we have the following fractional expression, where the numerator and denominator expressions can be computed following Table 2: $\overline{\mathrm{QSum}()}(\operatorname{Cube}(X))=\overline{\operatorname{QSum}(\operatorname{Cube}(X))} / \operatorname{Count}(\operatorname{Cube}(X))$.

We then consider $\left(\frac{\operatorname{Sum}()}{\operatorname{Count}()}\right)^{2}(\operatorname{Cube}(X))$. Based on Table 4 , we have

$$
\left(\frac{\operatorname{Sum}()}{\operatorname{Count}()}\right)^{2} \geq \operatorname{Min}\left(\underline{(\text { Sum } / \text { Count })^{+}} \times \underline{(\text { Sum } / \text { Count })^{+}},\right.
$$

Count is always positive. By following Table 5 for computing sign bounds, we get $\left(\frac{\operatorname{Sum}()}{\operatorname{Count}()}\right)^{2}(\operatorname{Cube}(X))$ :

8. There is another definition of Variance: $\operatorname{Var}(X)=\frac{\sum_{i}\left(x_{i}-\bar{X}\right)^{2}}{N-1}$. The discussions here also apply to this definition. 


$$
\begin{gathered}
\operatorname{Min}\left(\left(\underline{\operatorname{Sum}^{+}(\operatorname{Cube}(X))} / \overline{\operatorname{Count}(\operatorname{Cube}(X))}\right)^{2},\right. \\
\left.\left(\overline{\operatorname{Sum}^{-}(\operatorname{Cube}(X))} / \overline{\operatorname{Count}(\operatorname{Cube}(X))}\right)^{2}\right) .
\end{gathered}
$$

In the above equation,

$$
\overline{\operatorname{Count}(\operatorname{Cube}(X))}=\operatorname{Sum}_{i} \operatorname{Count}\left(X_{i}\right) \text {. }
$$

Moreover, based on Table 3,

$$
\underline{\operatorname{Sum}^{+}(\operatorname{Cube}(X))}=\operatorname{Min}_{i} \operatorname{Sum}\left(X_{i}\right)
$$

if $\operatorname{Sum}\left(X_{i}\right) \geq 0$ for all $i=1 \ldots n$; otherwise, it is 0 . Similarly, $\overline{\operatorname{Sum}^{-}(\operatorname{Cube}(X))}=\operatorname{Max} \operatorname{Sum}\left(X_{i}\right)$ if $\operatorname{Sum}\left(X_{i}\right)<$ 0 for all $i=1 \ldots n$; otherwise, it is 0 .

In summary, we compute $\overline{\operatorname{Var}(\operatorname{Cube}(X))}$ as follows:

$$
\begin{aligned}
& \frac{\operatorname{if} \operatorname{Sum}\left(X_{i}\right) \geq 0 \text { for } i=1 . . n,}{\underset{i}{\operatorname{Sum} \operatorname{Sum}\left(X_{i}^{2}\right)} / \operatorname{Min}_{i} \operatorname{Count}\left(X_{i}\right)} \\
& -\left(\underset{i}{\operatorname{Min} \operatorname{Sum}}\left(X_{i}\right) / \operatorname{Sum}_{i} \operatorname{Count}\left(X_{i}\right)\right)^{2} ; \\
& \frac{\text { otherwise, if } \operatorname{Sum}\left(X_{i}\right)<0 \text { for } i=1 . . n,}{\operatorname{Sum}_{i} \operatorname{Sum}\left(X_{i}^{2}\right) / \operatorname{Min}_{i} \operatorname{Count}\left(X_{i}\right)} \\
& -\left(\operatorname{Max}_{i} \operatorname{Sum}\left(X_{i}\right) / \operatorname{Sum}_{i} \operatorname{Count}\left(X_{i}\right)\right)^{2} ;
\end{aligned}
$$

otherwise,

$$
\overline{\operatorname{Sum}} \operatorname{Sum}_{i}\left(X_{i}^{2}\right) / \operatorname{Min}_{i} \operatorname{Count}\left(X_{i}\right) \text {. }
$$

With these equations, obviously tighter $\overline{\operatorname{Var}(\operatorname{Cube}(X))}$ is obtained if the sums of MSPs are of the same sign.

\section{Optimization for Bounding Complex FUNCTIONS}

When rewriting several commonly used algebraic aggregation functions, namely, Avg, Var, and Standard-Deviation, into arithmetic expressions of distributive aggregate functions, we notice that very often, they use the following subexpression:

$$
F(X)=\operatorname{Sum}_{i} G_{1}\left(X_{i}\right) / \operatorname{Sum}_{i} G_{2}\left(X_{i}\right),
$$

where $G_{1}$ and $G_{2}$ are some distributive aggregate functions, and $X_{1}, \ldots, X_{n}$ are MSPs. (We can rewrite $\operatorname{Avg}(X)$ as $\operatorname{Sum}(X) / \operatorname{Count}(X)$ and $\operatorname{Var}(X)$ as

$$
\operatorname{QSum}(X) / \operatorname{Count}(X)-(\operatorname{Sum}(X) / \operatorname{Count}(X))^{2} .
$$

Moreover, we have

$$
\operatorname{Sum}(X) / \operatorname{Count}(X)=\operatorname{Sum}_{i} \operatorname{Sum}\left(X_{i}\right) / \operatorname{Sum}_{i} \operatorname{Count}\left(X_{i}\right),
$$

which is $F$, with $G_{1}=$ Sum and $G_{2}=$ Count.) Therefore, it is desirable to give tighter bounds for $F$ than those obtained by Table 4 . The next result provides not only tight bounds but also the optimal.

Theorem 3. Let $\operatorname{Cube}(X)$ be a data cube over measure $X$ with MSPS $X_{1}, \ldots, X_{n}$, let $G_{1}$ and $G_{2}$ be aggregate functions, and let $F$ be as defined above. If $G_{2}\left(X_{i}\right)>0$ for all $i=1 \ldots n$, then we can bound $F$ using

$$
\overline{F(\operatorname{Cube}(X))}=\operatorname{Max}_{i} F\left(X_{i}\right), \underline{F(\operatorname{Cube}(X))}=\operatorname{Min}_{i} F\left(X_{i}\right) .
$$

Moreover, these bounds are the optimal bounds for $F(\operatorname{Cube}(X))$.

Proof. Without loss of generality, we can assume that $\operatorname{Min} F\left(X_{i}\right)=F\left(X_{1}\right)$, and $\operatorname{Max} F\left(X_{i}\right)=F\left(X_{n}\right)$. Given a group $g$ of $\operatorname{Cube}(X)$, let ${ }^{i} X_{g_{1}}, \ldots, X_{g_{k}}$ be the MSPs contained in $g$. Thus, $g=X_{g_{1}} \cup \ldots \cup X_{g_{k}}$. Then,

$$
\begin{aligned}
F(g) & =\operatorname{Sum}_{j} G_{1}\left(X_{g_{j}}\right) / \operatorname{Sum}_{j} G_{2}\left(X_{g_{j}}\right), \\
& =\frac{G_{1}\left(X_{g_{1}}\right)+\ldots+G_{1}\left(X_{g_{k}}\right)}{G_{2}\left(X_{g_{1}}\right)++G_{2}\left(X_{g_{k}}\right)}, \\
& =\frac{F\left(X_{g_{1}}\right) \times G_{2}\left(X_{g_{1}}\right)+\ldots+F\left(X_{g_{k}}\right) \times G_{2}\left(X_{g_{k}}\right)}{G_{2}\left(X_{g_{1}}\right)+\ldots+G_{2}\left(X_{g_{k}}\right)} .
\end{aligned}
$$

The division in the last step is permitted, since $G_{2}\left(X_{g_{i}}\right)>$ 0 for all $i$. Therefore,

$$
\begin{aligned}
& F(g)-F\left(X_{n}\right) \\
= & \frac{F\left(X_{g_{1}}\right) \times G_{2}\left(X_{g_{1}}\right)+\ldots+F\left(X_{g_{k}}\right) \times G_{2}\left(X_{g_{k}}\right)}{G_{2}\left(X_{g_{1}}\right)+\ldots+G_{2}\left(X_{g_{k}}\right)} \\
& -F\left(X_{n}\right) \times \frac{G_{2}\left(X_{g_{1}}\right)+\ldots+G_{2}\left(X_{g_{k}}\right)}{G_{2}\left(X_{g_{1}}\right)+\ldots+G_{2}\left(X_{g_{k}}\right)} \\
= & \frac{\left(F\left(X_{g_{1}}\right)-F\left(X_{n}\right)\right) \times G_{2}\left(X_{g_{1}}\right) \ldots+\left(F\left(X_{g_{k}}\right)-F\left(X_{n}\right)\right) \times G_{2}\left(X_{g_{k}}\right)}{G_{2}\left(X_{g_{1}}\right)+\ldots+G_{2}\left(X_{g_{k}}\right)} .
\end{aligned}
$$

Since $F\left(X_{g_{j}}\right) \leq F\left(X_{n}\right)$ and $G_{2}\left(X_{g_{j}}\right)>0$ for all $j=1 \ldots k$, we have $G_{2}\left(X_{g_{1}}\right)+\ldots+G_{2}\left(X_{g_{k}}\right)>0$ and $\left(F\left(X_{g_{j}}\right)-\right.$ $\left.F\left(X_{n}\right)\right) \times G_{2}\left(X_{g_{j}}\right) \leq 0$ for all $j$. Therefore, $F(g)-$ $F\left(X_{n}\right) \leq 0$ or, equivalently, $F(g) \leq F\left(X_{n}\right)$. Similarly, it can be shown that $F(g) \geq F\left(X_{1}\right)$. Thus, we have proven that $F\left(X_{n}\right)$ and $F\left(X_{1}\right)$ are the upper and lower bounds for $F(\operatorname{Cube}(X))$, respectively.

As $\operatorname{Min}_{i} F\left(X_{i}\right)$ is the smallest aggregate for an MSP and thus is a real aggregate in $F(\operatorname{Cube}(X))$, all other lower bounds for $F(\operatorname{Cube}(X))$ must be greater than $\operatorname{Min}_{i} F\left(X_{i}\right) . \operatorname{Min}_{i} F\left(X_{i}\right)$ is thus the tightest lower bound for $F(\operatorname{Cube}(X))$. Similarly, we can prove that $\operatorname{Max}_{i} F\left(X_{i}\right)$ is the tightest upper bound for $F(\operatorname{Cube}(X))$.

Next, we show that the bounds obtained by following Tables 2 and 4 are weaker. Considering $\operatorname{Max}_{i} F\left(X_{i}\right)$ (and still assuming that $\operatorname{Max}_{i} F\left(X_{i}\right)=F\left(X_{n}\right)$ ), the case for $\operatorname{Min}_{i} F\left(X_{i}\right)$ is similar. By following Tables 2 and 4, $\overline{F(\operatorname{Cube}(X))}$ is computed:

Case 1. There exists $j$ such that $G_{1}\left(X_{j}\right)>0$. $\overline{F(\operatorname{Cube}(X))}$ is

$\overline{\operatorname{Sum}_{i} G_{1}\left(X_{i}\right)} / \operatorname{Sum}_{i} G_{2}\left(X_{i}\right)=\operatorname{Sum}_{G_{1}\left(X_{i}\right) \geq 0} G_{1}\left(X_{i}\right) / \operatorname{Min}_{i} G_{2}\left(X_{i}\right)$.

Since $G_{2}\left(X_{i}\right)>0$ for all $i=1 \ldots n$, and $G_{1}\left(X_{j}\right)>0$, $F\left(X_{j}\right)>0$. Since $\operatorname{Max}_{i} F\left(X_{i}\right)=F\left(X_{n}\right)$, it follows that $G_{1}\left(X_{n}\right)>0$. Since $\operatorname{Sum}_{G_{1}\left(X_{i}\right) \geq 0} G_{1}\left(X_{i}\right) \geq G_{1}\left(X_{n}\right)$ and $\operatorname{Min}_{i} G_{2}\left(X_{i}\right) \leq G_{2}\left(X_{n}\right)$, we have

$$
\operatorname{Sum}_{G_{1}\left(X_{i}\right) \geq 0} G_{1}\left(X_{i}\right) / \operatorname{Min}_{i} G_{2}\left(X_{i}\right) \geq \frac{G_{1}\left(X_{n}\right)}{G_{2}\left(X_{n}\right)}=F\left(X_{n}\right) .
$$


TABLE 6

Bounding Complex Aggregate Functions

\begin{tabular}{l}
$\overline{\overline{\operatorname{Avg}(\operatorname{Cube}(X))}}=\operatorname{Max}_{i} \operatorname{Avg}\left(X_{i}\right), \underline{\operatorname{Avg}(\operatorname{Cube}(X))}=\operatorname{Min}_{i} \operatorname{Avg}\left(X_{i}\right)$ \\
\hline if there exist $i$ and $j$ such that $\operatorname{Sum}\left(X_{i}\right)$ is positive and $\operatorname{Sum}\left(X_{j}\right)$ is negative, \\
$\overline{\operatorname{Var}(\operatorname{Cube}(X))}=\operatorname{Max}_{i} \frac{\operatorname{Sum}\left(X_{i}^{2}\right)}{\operatorname{Count}\left(X_{i}\right)}$, \\
$\overline{\operatorname{Var}(\operatorname{Cube}(X))}=\operatorname{Min}_{i} \frac{\operatorname{Sum}\left(X_{i}^{2}\right)}{\operatorname{Count}\left(X_{i}\right)}-\operatorname{Max}\left(\left(\operatorname{Max}_{i} \operatorname{Avg}\left(X_{i}\right)\right)^{2},\left(\operatorname{Min}_{i} \operatorname{Avg}\left(X_{i}\right)\right)^{2}\right) ;$ \\
if $\operatorname{Sum}\left(X_{i}\right) \geq 0$ for all $i(1 \leq n)$ \\
$\overline{\operatorname{Var}(\operatorname{Cube}(X))}=\operatorname{Max}_{i} \frac{\operatorname{Sum}\left(X_{i}^{2}\right)}{\operatorname{Count}\left(X_{i}\right)}-\left(\operatorname{Min}_{i} \operatorname{Avg}\left(X_{i}\right)\right)^{2}$, \\
$\underline{\operatorname{Var}(\operatorname{Cube}(X))}=\operatorname{Min}_{i} \frac{\operatorname{Sum}\left(X_{i}^{2}\right)}{\operatorname{Count}\left(X_{i}\right)}-\left(\operatorname{Max}_{i} \operatorname{Avg}\left(X_{i}\right)\right)^{2} ;$ \\
if $\operatorname{Sum}\left(X_{i}\right) \leq 0$ for for all $i(1 \leq i \leq n)$ \\
$\overline{\operatorname{Var}(\operatorname{Cube}(X))}=\operatorname{Max}_{i} \frac{\operatorname{Sum}\left(X_{i}^{2}\right)}{\operatorname{Count}\left(X_{i}\right)}-\left(\operatorname{Max}_{i} \operatorname{Avg}\left(X_{i}\right)\right)^{2}$, \\
$\underline{\operatorname{Var}(\operatorname{Cube}(X))}=\operatorname{Min}_{i} \frac{\operatorname{Sum}\left(X_{i}^{2}\right)}{\operatorname{Count}\left(X_{i}\right)}-\left(\operatorname{Min}_{i} \operatorname{Avg}\left(X_{i}\right)\right)^{2}$.
\end{tabular}

Cube $(X)$ is a data cube on measure $X$ with MSPs $\left\{X_{1}, \ldots, X_{n}\right\}$.

Hence, $\operatorname{Max}_{i} F\left(X_{i}\right)=F\left(X_{n}\right)$ is a tighter bound.

Case 2. For all $i(1 \leq i \leq n), G_{1}\left(X_{i}\right) \leq 0 . \overline{F(\operatorname{Cube}(X))}$ is

$\overline{\operatorname{Sum}_{i} G_{1}\left(X_{i}\right)} / \overline{\operatorname{Sum}_{i} G_{2}\left(X_{i}\right)}=\operatorname{Max}_{i} G_{1}\left(X_{i}\right) / \operatorname{Max}_{i} G_{2}\left(X_{i}\right)$.

As

$$
0 \geq \operatorname{Max}_{i} G_{1}\left(X_{i}\right) \geq G_{1}\left(X_{n}\right)
$$

and $0<G_{2}\left(X_{n}\right) \leq \operatorname{Max}_{i} G_{2}\left(X_{i}\right)$,

$0 \geq \operatorname{Max}_{i} G_{1}\left(X_{i}\right) / \operatorname{Max}_{i} G_{2}\left(X_{i}\right) \geq G_{1}\left(X_{n}\right) / G_{2}\left(X_{n}\right)=F\left(X_{n}\right)$.

Hence, $\operatorname{Max}_{i} F\left(X_{i}\right)=F\left(X_{n}\right)$ is a tighter bound.

By following Theorem 3, tighter bounds for Avg and Var can be computed, as shown in Table 6 .

In Section 7, we present an efficient implementation of the BP-Cubing algorithm, utilizing the bounding results discussed so far.

\section{Bound-Prune Cubing Algorithms}

We first present the group tree (G-tree) data structure that we will use for iceberg cubing. We then explain how bound prune cubing (BP-Cubing) is implemented on the G-tree. Finally, we briefly discuss how our algorithms utilize antimonotone constraints. The iceberg cube with the constraint "Avg(Sale) in $[15,20]$ " on the Sales data set in Table 1 is used as a running example throughout this section.

\subsection{The G-Tree}

The underlying data structure for BP-Cubing is the G-tree. A G-tree is the compression of a given input data set and is constructed by one scan of the data set. It is used for both the top-down and bottom-up bound prune cubing algorithms (BP-Cubing(TD) and BP-Cubing(BU), respectively), although there are some minor differences depending on the traversal strategy.

The G-tree for the data set in Table 1 is shown in Fig. 3. A G-tree for an $n$-dimensional data set is of depth $n$, where each level represents a dimension. A path starting from the root collapses the tuples with common dimension values along the path. Each tree node keeps

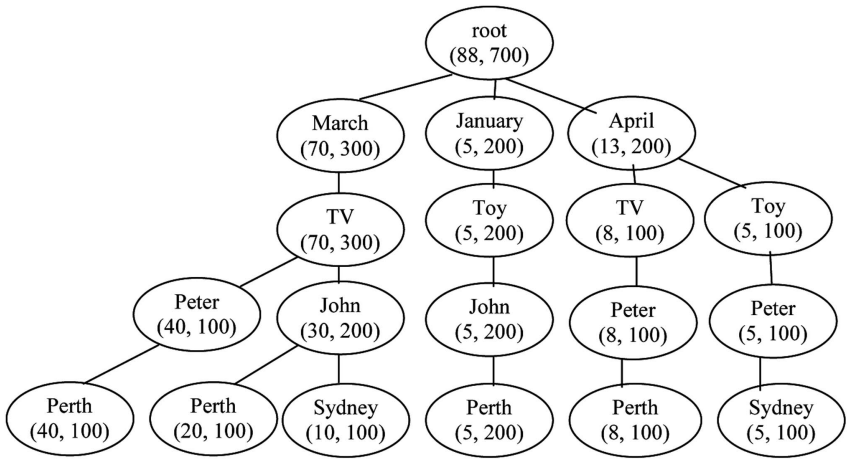

Fig. 3. A G-tree for the data set in Table 1.

the auxiliary aggregates necessary to compute the iceberg cube. In our example, the auxiliary aggregates in a node are Sum (Sale) and Count $(*)$. For the leftmost path from the root of the G-tree in Fig. 3, the node (March) shows that there are 70 tuples with $\operatorname{Sum}(\mathrm{Sale})=300$ in the (March, $*, *, *)$ partition, whereas the node (Peter) shows that there are 40 tuples with $\operatorname{Sum}(\mathrm{Sale})=100$ in the (March, TV, Peter, $*$ ) partition.

For a given data set, different dimension orders result in different G-trees. Depending on whether the G-tree is traversed top-down or bottom-up in computing cubes, the tree should be constructed in different orders of dimensions. Antimonotone pruning is effective when the most discriminating dimension is examined first. This suggests the cardinality-descending order of dimensions during cube computation. As a result, for bottom-up traversal, the cardinality-ascending order should be used in constructing the G-tree. In contrast, for top-down traversal, the cardinality-descending order should be used in constructing the G-tree. Our experiments have confirmed that such heuristics indeed have a positive impact on the effectiveness of pruning and the efficiency of cubing algorithms.

\subsection{Top-Down Bound-Prune Cubing on G-Trees}

An observation of Fig. 3 reveals some useful facts: The path from the root to a node of the G-tree aggregates a group, and each level of the G-tree computes a group-by of the cube lattice. For the G-tree in Fig. 3, the root node has the aggregate for the group $(*, *, *, *)$, with $\operatorname{Count}(*)=88$, and $\operatorname{Sum}(\mathrm{Sale})=700$. The nodes at level 1 compute the aggregates for groups in (Month, $*, *, *)$, which are (March, $*, *, *, 70,300)$, (January, $*, *, *, 5,200)$, and (April, $*, *, *, 13,200)$. The nodes at the next three levels compute the aggregates for the group-bys (Month, Product), (Month, Product, SalesMan), and (Month, Product, SalesMan, City), respectively. The leaf nodes give the MSPs for Cube (Month, Product, SalesMan, City). We summarize these in the following observations.

Observation 2. In a G-tree, the aggregates in each node are the aggregates for the group with dimension values on the path from the root to the node.

Observation 3. In a G-tree, for $n$ dimensions $A_{1}, \ldots, A_{n}$, the leaf nodes are the MSPs for $\operatorname{Cube}\left(A_{1}, \ldots, A_{n}\right)$. 


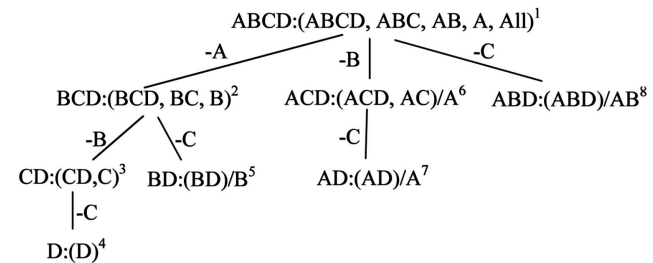

Fig. 4. Top-down bound prune cubing of $\mathrm{Cube}(A B C D)$ : For each node, we show a G-tree (before ":"), the group-bys (after ":") computed in the G-tree, and the shared dimensions (after the ""). The numbers show the order in which trees are built.

G-trees are constructed to compute all group-bys in a data cube. When constructing a G-tree for an $n$-dimensional data set, we simultaneously compute $n$ group-bys, namely, the group-bys whose dimensions are prefixes of the list of dimensions ordered by the levels of the tree. To compute the other group-bys in the cube, we collapse one dimension from a given G-tree at a time to construct a sub-G-tree ${ }^{9}$ and compute the corresponding group-bys of the sub-G-tree. For example, by collapsing dimension Product in the original G-tree, given in Fig. 3, we get the subtree \{Month, (-Product), SalesMan, City, Customer\}, shown in Fig. 5.

Fig. 4 shows the (sub)G-trees constructed for computing the data cube on dimensions A, B, C, and D. Each node in Fig. 4 represents a G-tree and the set of all corresponding group-bys. The $A B C D$ tree at the top is constructed by one scan of the data set, and the corresponding group-bys ( $A$, $B, C, D)$, which are the $(A, B, C),(A, B),(A)$, and () trees are computed during this scan. The sub-G-trees of the $A B C D$ tree, which are the $(-A) B C D, A(-B) C D$, and $A B(-C) D$ trees, are formed by collapsing dimensions $\mathrm{A}, \mathrm{B}$, and $\mathrm{C}$, respectively. The $C D$ tree is a subtree of the $B C D$ tree and recursively is also a subtree of the $A B C D$ tree. The dimensions after "/" in the nodes denote common prefix dimensions for the tree at the node and all of its subtrees. A is the common prefix dimension for the $A C D$ tree and its subtrees. All group-bys that are computed on the ACD tree and its subtrees form subcubes for $A$ values Cube(CD) $\mid a_{i}$. The leaf nodes originating from $a_{i}$ are the MSPs for Cube(CD) $\mid a_{\mathrm{i}}$. In the sub-G-tree construction process, we also compute the bounds and use them for pruning, as described in the observation below.

Observation 4. With top-down aggregation, given a G-tree $G$ with $n$ dimensions $A_{1}, \ldots, A_{n}$ and a subtree $G_{k}$, by collapsing a dimension $A_{k}, 1<\mathrm{k}<\mathrm{n}, A_{1}, \ldots, A_{k-1}$ are the common prefix dimensions for $G_{k}$ and all its subtrees. Each node $\left(a_{1}, \ldots, a_{k-1}\right)$ of $G$ gives the prefix dimension values for Cube $\left(A_{k+1}, \ldots, A_{n}\right) \mid a_{1}, \ldots, a_{k-1}$. The core of the cube consists of the MSPs corresponding to the leaf nodes of the branches originating from the node $\left(a_{1}, \ldots, a_{k-1}\right)$. If the bounds of $\operatorname{Cube}\left(A_{k+1}, \ldots, A_{n}\right) \mid a_{1}, \ldots, a_{k-1}$ fail the given constraint, then those branches can be pruned.

Example 9. Consider the G-tree $G$ in Fig. 3 as the original tree and the G-tree $G_{p}$ in Fig. 5 as the subtree. Month is the prefix dimension for the group-bys on $G_{p}$ and the subtree of $G_{p}$. The subcubes are

9. It should be pointed out that a sub-G-tree is not part of an original G-tree but is obtained by collapsing a given dimension of the original G-tree.

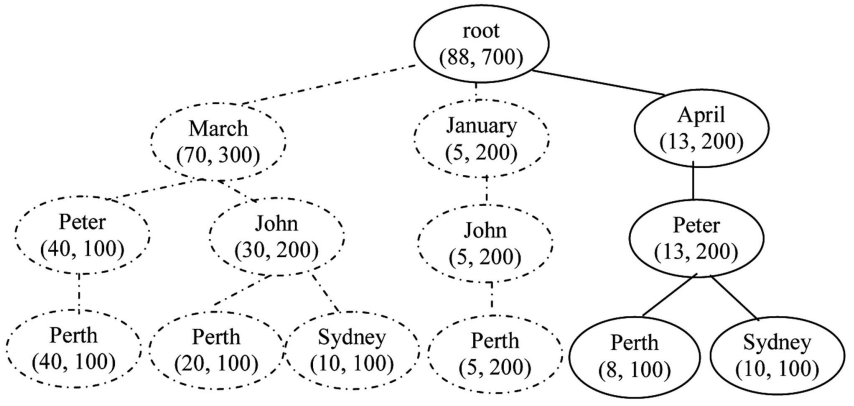

Fig. 5. The subtree on $\{$ Month, (-Product), SalesMan, City $\}$ obtained by collapsing dimension Product in the original G-tree, given in Fig. 3.

1. Cube(SalesMan, City)|March,

2. Cube(SalesMan, City)|January, and

3. Cube(SalesMan, City)|April.

In $G$, the leaf nodes of the March subtree are the MSPs of Cube(SalesMan, City)|March. With the constraint "Avg (Sale) in [15, 20]," $G$ is pruned in constructing the subtree $G_{p}$. By following Table 6, the bounds for Cube(SalesMan, City)|March are computed from the three leaf nodes originating from node (March) of $G$ :

$$
\begin{aligned}
\overline{\operatorname{Avg}(\text { Cube }(\text { Sale }) \mid \operatorname{Mar})} & =\operatorname{Max}(\{100 / 40,100 / 20,100 / 10\}) \\
& =10 . \\
\underline{\operatorname{Avg}(\text { Cube }(\text { Sale }) \mid \operatorname{Mar})} & =\operatorname{Min}(\{100 / 40,100 / 20,100 / 10\}) \\
& =2.5 .
\end{aligned}
$$

As $[2.5,10]$ violates "Avg (Sale) in [15, 20]," all three branches originating from (March) are removed from further computation. Similarly, Avg(Sale) of Cube(Salesman, City)|January is bounded as [40, 40], which violates the constraint, and is pruned. Avg ( $\mathrm{Sale}$ ) of Cube(Salesman, City)|April is bounded as [12.5, 25], which does not violate the constraint, and the branches from (April) are kept in $G_{p}$. The nodes in dashed lines in Fig. 5 highlight the fact that the branches from (March) and (January) are pruned before the $G_{p}$ tree is generated, and importantly, they are permanently pruned from all future recursive computation.

\subsection{The Top-Down Bound-Prune Cubing Algorithm}

In this section, we present the Top-Down Bound-Prune Cubing (BP-Cubing(TD)) algorithm, shown in Algorithm 1, for computing iceberg data cubes. It performs boundpruning in the top-down multiway aggregation manner, and it uses the G-trees.

\section{Algorithm 1. The Top-Down Bound-Prune Cubing Algorithm}

Input: A data set $D$ over dimensions $A_{1}, \ldots, A_{n}$ and aggregation constraint $C$, assumed global.

Output: An $n$-dimensional iceberg cube on $D$ satisfying $C$.

(1) Build the G-tree $T\left(A_{1}, \ldots, A_{n}\right)$ from $D$;

(2) Output aggregates satisfying $C$, computed when $T$ was built;

(3) for $i=1 \ldots(n-1)$, do

(4) $\quad$ BP-Cubing $\left(T\left(A_{1}, \ldots, A_{n}\right), A_{i}\right)$; 


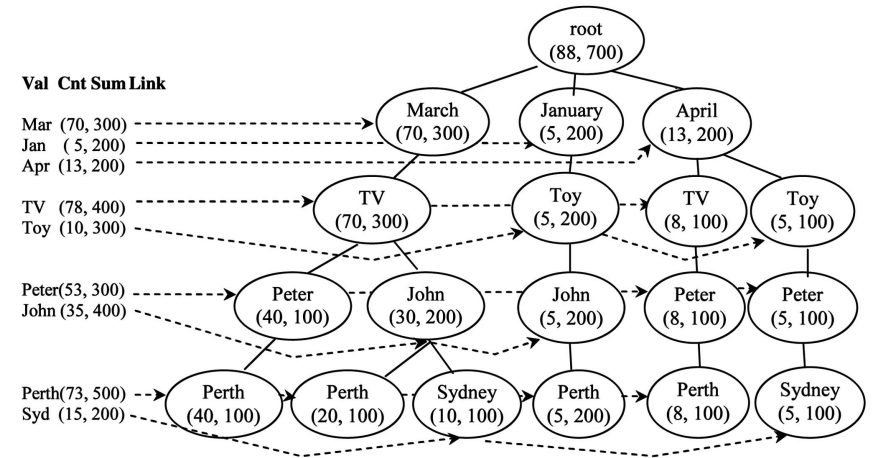

Fig. 6. The G-tree for the data set in Table 1, with header table and side links.

// $B_{i}$, the ith dimension of $T$ is the dimension to be collapsed Procedure BP-Cubing $\left(T\left(B_{1}, \ldots, B_{k}\right), B_{i}\right)$

(5) $T_{s} \leftarrow$ nil;

//construct subtree $T_{s}$ by collapsing $B_{i}$.

(6) for each node $n_{i-1}$ of dimension $B_{i-1}$ in $T$, do

(7) $\quad V_{i-1} \leftarrow$ dimension values on the path from root to $n_{i-1}$;

// the MSPs for $\operatorname{Cube}\left(B_{i+1}, \ldots, B_{k}\right) \mid V_{i-1}$

(8) Let $M$ be the set of leaves originating from $n_{i-1}$;

(9) Compute the bounds for $\operatorname{Cube}\left(B_{i+1}, \ldots, B_{k}\right) \mid V_{i-1}$ from $M$; // prune the paths if the bounds violate $C$ if the bounds do not violate $C$ then

Collapse $B_{i}$ and add the paths passing $n_{i-1}$ of $T$ to $T_{s}$;

(12) Output aggregates on $T_{s}$ that satisfy $C$;

(13) for $j=(i+1) \ldots(k-1)$ do

(14) BP-Cubing $\left(T_{s}\left(B_{1}, \ldots, B_{i-1}, B_{i+1}, \ldots, B_{k}\right), B_{j}\right)$

Let $D$ be a data set with $n$ dimensions $A_{1}, \ldots, A_{n}$ and $C$ be the iceberg constraint. To compute the iceberg cube, first, a G-tree $T$ is built by one scan of $D$ (line 1). As mentioned in Observation 2, we simultaneously aggregate $n$ group-bys, namely, $\left(A_{1}\right),\left(A_{1}, A_{2}\right), \ldots,\left(A_{1}, A_{2}, \ldots, A_{n}\right)$, when building the G-tree. Those groups that satisfy $C$ are output (line 2).

The Bound-Prune Cubing (BP-Cubing) procedure computes the iceberg cube by recursively collapsing dimensions and building sub-G-trees. Given a G-tree, before a subtree is built, bounds for branches are computed, and those branches whose bounds fail the constraint are pruned from further computation (lines 9-11). The leaf nodes of a G-tree are the MSPs for the G-tree, and these MSPs are used to compute the corresponding aggregate bounds. $T_{s}$ is a subtree of $T$, obtained by collapsing dimension $B_{i}$; the collapsing computation is described in lines 6-11. Aggregates are computed during the construction of $T_{s}$. Then, BPCubing is recursively called (lines 13-14), where group-bys of dimensions after $B_{i}$ are computed.

\subsection{Bottom-Up Bound-Prune Cubing on G-Trees}

The BP-Cubing(BU) algorithm is similar to Algorithm 1. With the bottom-up cubing strategy, a group is computed before its subgroups. There are several differences concerning 1) the G-tree used, 2) how sub-G-trees are constructed, and 3) how bounding with MSPs are done. We describe these below.

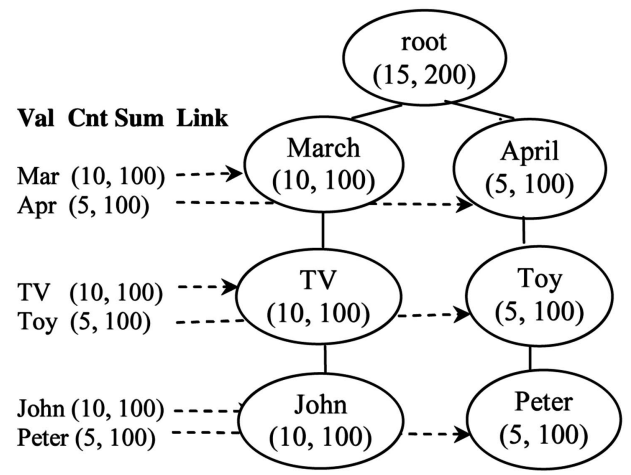

Fig. 7. The subtree of the G-tree in Fig. 6, with City = Sydney.

We first consider the G-tree used. In BUC, a G-tree will also contain a header table and side links. A header table entry represents a one-dimensional group together with the corresponding aggregates, and it is linked to nodes with the corresponding dimension value. Fig. 6 shows such a G-tree for the sample Sales data set. The side-links will be used to efficiently derive aggregates of other groups in subsequent computation.

We now consider how sub-G-trees are constructed. In the first G-tree for a data set, the header table contains onedimensional groups. In the sub-G-trees constructed from a given G-tree, the header table contains groups whose dimensionality is one level higher than that of groups for the given G-tree. For the $G$-tree in Fig. 6, we build sub-Gtrees for the 2D subgroups of the (City) groups, namely, the groups of (Salesman, City), (Product, City), and (Month, City). Similarly, we build sub-G-trees for the 3D subgroups of the (Salesman, City) groups, and so on. The sub-G-trees are obtained by merging paths in the original G-tree. For example, to compute the 2D subgroups of Sydney, the subtree in Fig. 7 is constructed by merging the paths from the root to the nodes on the Sydney side link. In this process, the aggregates for the nodes above the (Sydney) nodes need to be modified to remove the contribution of the non-Sydney cities. For example, the node (John) has the aggregates of $(30,200)$ for both Perth and Sydney but only has the aggregates of $(10,100)$ for Sydney.

We now turn to bounding with MSPs. Recall that, with the top-down aggregation strategy, all MSPs for a (sub) data cube are "conveniently available" for bounding (by "conveniently available," we mean that they are available without extra overhead). In the bottom-up aggregation, the "conveniently available" MSPs are those pointed to by side links of certain header table entries. The observation below describes how bounds are computed, and pruning is achieved.

Observation 5. For a G-tree of dimensions $A_{1}, \ldots, A_{n}$, the leaf nodes are the MSPS for $\operatorname{Cube}\left(A_{1}, \ldots, A_{n}\right)$. With the BUC strategy, for $a_{k} \in \operatorname{domain}\left(A_{k}\right)(1 \leq k \leq n)$, the subgroups of $\left(a_{k}\right)$ to be computed from the branches on the side link of $a_{k}$ comprise Cube $\left(A_{1}, \ldots, A_{k-1}\right) \mid a_{k}$, and MSPs of the cube are the leaf nodes on the side link for $a_{k}$ (available for bounding). The subtrees from the side link of $a_{k}$ can be pruned if the bounds of $\operatorname{Cube}\left(A_{1}, \ldots, A_{k-1}\right) \mid a_{k}$ fail the given constraint.

Example 10. With Cube(Month, Product, Salesman)|Sydney, shown in Fig. 6, its MSPs are the leaf nodes (Sydney, 10,100 ) and (Sydney, 5, 100) on the side link for sydney. Thus, 


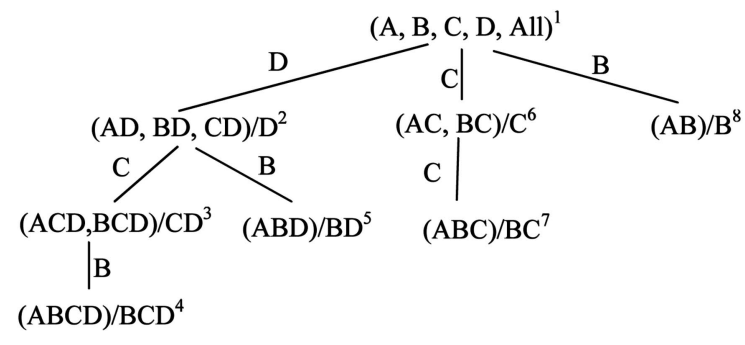

Fig. 8. Bottom-up bound prune cubing (BP-Cubing) on four dimensions $\mathrm{A}, \mathrm{B}, \mathrm{C}$, and $\mathrm{D}$

$\overline{\text { Cube(Month, Product, Salesman) } \mid \text { Sydney }}=100 / 5=20$,

$\overline{\text { Cube(Month, Product, Salesman) } \mid \text { Sydney }}=100 / 10=10$.

As $[10,20]$ does not violate the constraint "Avg (Sale) in $[15,20]$," the subtree for Sydney (shown in Fig. 7) is thus created.

In Fig. 8, we use an example to give some high-level view of the BUC process for a four-dimensional data cube. Each node represents the group that is computed on some G-tree. From top to bottom, nodes are linked by the subtree relationship. The superscripts over the nodes indicate the order in which the G-trees are created. With a tree, the dimensions after "/" are the shared dimensions for the tree and all its subtrees. They are also the conditional dimensions where the tree is created. Generally, the BP-Cubing(BU) algorithm is given in Algorithm 2.

Algorithm 2. The Bottom-Up Bound-Prune Cubing Algorithm

Input: A data set $D$ over dimensions $A_{1}, \ldots, A_{n}$ and aggregation constraint $C$.

Output: The $n$-dimensional iceberg cube on $D$, satisfying $C$.

(1) Build the G-tree $T\left(A_{1}, \ldots, A_{n}\right)$ from $D$;

(2) Output all aggregates in $T\left(A_{1}, \ldots, A_{n}\right)$.Header;

(3) foreach $a \in T$.Header, do

BP-Cubing $\left(T\left(A_{1}, \ldots, A_{n}\right),\{a\}\right)$;

Procedure BP-Cubing $\left(T\left(B_{1}, \ldots, B_{k}\right), S\right)$

/ / $S$ is a set of dimension values as the condition for subcubes.

(5) $T_{s}=$ nil;

(6) Suppose $a$ is on the $i^{\text {th }}$ dimension of $T$;

(7) Let $M$ be the nodes following the side link of $a$;

(8) Compute the bounds for $\operatorname{Cube}\left(B_{1}, \ldots, B_{i-1}\right) \mid S$

from the leaves originating from $M$;

(9) if the bounds do not violate $C$, then // pruning // Section 7.4

(10) Construct the subtree $T_{s}\left(B_{1}, \ldots, B_{i-1}\right)$;

(11) Output aggregates on $T_{s}$.Header that satisfy $C$;

(12) foreach $a_{s} \in T_{s}$.Header do

(13) $\quad$ BP-Cubing $\left(T_{s}\left(B_{1}, \ldots, B_{i-1}\right), S \cup\left\{a_{s}\right\}\right)$;

\subsection{Interaction with Antimonotone Constraints}

Our discussions have focused on complex nonantimonotone aggregation constraints. Antimonotone constraints can be easily incorporated in BP-Cubing as follows: Suppose an antimonotone constraint $C^{\prime}$ is present, in addition to the nonantimonotone constraint $C$. First, in lines 2 and 12 of Algorithm 1, a group $g$ is checked against both $C$ and $C^{\prime}$
TABLE 7

Census, TPC-R, and Weather Multidimensional Data Sets

\begin{tabular}{llll}
\hline Dataset & Cardinality(Range: Average) & \# Dimensions & \# records \\
\hline Census & {$[7 . .48]: 14$} & 12 & 88,443 \\
TPC-R & {$[3 . .25]: 10$} & 8 & $10^{6}$ \\
Weather & {$[2 . .6505]: 812$} & 9 & $10^{5}$ \\
\hline
\end{tabular}

before being output. More importantly, before the bounds are computed at line 9 , the branches of $T$ originating from $n_{i}$ are pruned from further computation if $n_{i}$ fails $C^{\prime}$, as all subgroups of $n_{i}$ will also fail $C^{\prime}$. With top-down cubing, the prefix groups before the collapsing dimension that fail $C^{\prime}$ are pruned. With bottom-up cubing, header-table entries that fail $C^{\prime}$ are pruned from further computation.

\section{EXPERIMENTS}

In this section, we evaluate the performance of both the BPCubing(TD) and BP-Cubing(BU) algorithms with experiments. We compare the performance of these algorithms with that of the DnA algorithm [14], [15] on non-antimonotone aggregation constraints and also with that of the BUC algorithm [4] on antimonotone constraints.

DnA is recent work on pruning for nonantimonotone aggregation constraints. As DnA is not designed for pruning for antimonotone constraints, extra processing is needed to prune for such constraints. On the other hand, BUC uses the same partition-based bottom-up aggregation strategy as DnA and is designed for antimonotone constraints. Thus, BP-Cubing is compared with BUC on pruning with antimonotone constraints.

To do fair comparison, all algorithms were implemented with all possible optimization techniques. BUC was implemented with the collapsing duplicates optimization [4]. We added collapsing duplicates and indexing tuples to DnA, even though such optimizations were not reported in the original papers [14], [15]. Block memory allocation is used in the BP-cubing algorithms to reduce the number of calls of the dynamic memory allocation functions. It is assumed that, for all algorithms, data structures used can fit into memory. All experiments were performed on a PC with an i686 processor running GNU/Linux. As outputting the groups can take a significant amount of time for big data cubes, we choose to exclude the time for output in the timing for the algorithms. The thresholds for constraints are selected in a way such that there are at least 10 groups in the output. We experimented with constraints involving the aggregate functions Count, Avg, Var, and Sum.

\subsection{Data Sets}

We used real-world, as well as artificial, data sets in our experiments. When selecting the data sets, we considered the following data characteristics: dense versus sparse and random versus skewed. Table 7 summarizes the data sets used in our experiments.

The US census data set ${ }^{10}$ was collected in a 1990 US households survey. The original data set had 61 attributes such as hrswork1 (hours worked last week), nchild (number of own children on the household), and valueh (value of house). We selected 12 discrete attributes as

10. ftp://ftp.ipums.org/ipums/data/ip19001.Z. 


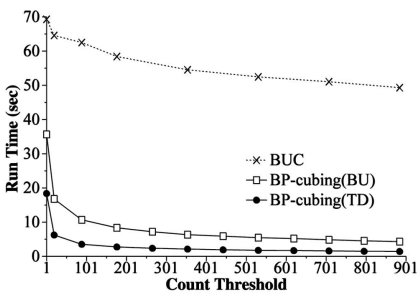

(a)

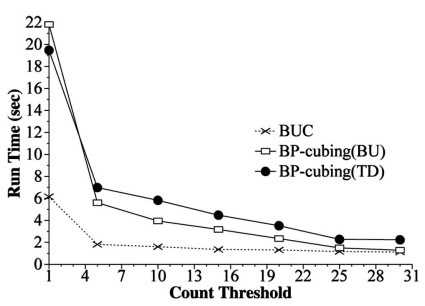

(c)

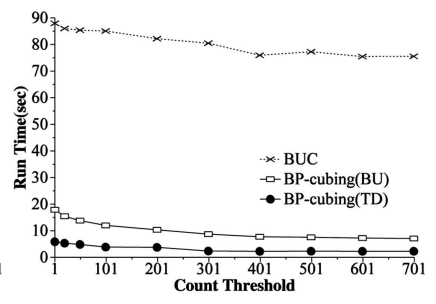

(b)

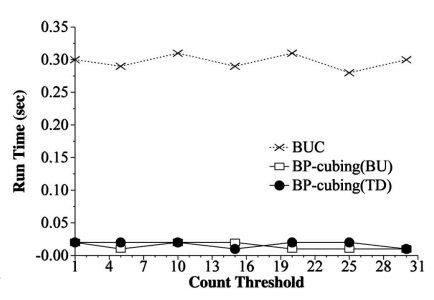

(d)
Fig. 9. BP-Cubing versus BUC on the "Count $(*) \geq \alpha$ " constraint. (a) Census. (b) TPC-R. (c) Weather. (d) Weather-5.

dimensions and a numerical attribute as the measure. The data set is dense and skewed.

The Weather data set ${ }^{11}$ contains real weather reports from various weather stations in 1985. We used these nine attributes as dimensions: station-id, longitude, solar-altitude, latitude, present-weather, weather-change-code, day, hour, and brightness. Their cardinalities, respectively, are 6,505, 351, 179, 152, $99,10,8,3$, and 2 . We randomly generated values between 1 and 100 as the measure. This Weather data set is the same as that used in the experiments in [4], where BUC was shown to be efficient for sparse data.

The TPC-R data set ${ }^{12}$ is an artificial data set provided by the Transaction Processing Council and is designed for testing the performance of representative complex queries in high-level business decision-making environment. Its dimensions include customer, supplier, order, and shipment. The original TPC-R data set consists of several relational tables. We constructed a joined relation as our multidimensional data set. A numerical attribute is selected as the measure. The TPC-R data set is relatively dense and random.

\subsection{BP-Cubing versus BUC on "Count $(*) \geq \alpha$ "}

This set of experiments is designed to evaluate two aspects of BP-Cubing: the tree-based aggregation strategy and pruning with simple antimonotone constraints. This involves comparing the performance of the BP-Cubing algorithms with BUC on computing iceberg cubes for the constraint "Count $(*) \geq \alpha$." For the special case of $\alpha=1$, the full data cubes are computed. Since there is no pruning, the difference in efficiency should be solely attributed to the aggregation strategies. Fig. 9 shows the runtime of the algorithms.

Fig. 9a shows that BUC uses 69.31 seconds to compute the full data cube over the dense and skewed Census data set, and BP-Cubing(BU) and BP-Cubing(TD) take 35.67 and 18.39 seconds, respectively, which are 1.9 and 3.76 times

11. http:/ /cdiac.ornl.gov/ftp/ndp026b/SEP85L.DAT.Z. 12. http://www.tpc.org/tpcr/. faster than BUC. Fig. 9b shows that BUC uses 87.97 seconds to compute the full cube for the TPC-R data set. In contrast, BP-Cubing(BU) and BP-Cubing(TD) use only 17.9 and 5.89 seconds, respectively, which are 4.9 and 14.9 times faster. On the other hand, BUC is more efficient on the sparse Weather data than the BP-Cubing algorithms. Fig. 9c shows that BUC uses 6.16 seconds on computing the full cube, whereas BP-Cubing(BU) and BP-Cubing(TD) use 21.82 and 19.48 seconds, respectively. When executed on the data set obtained by removing the four dimensions with the largest cardinalities of 6,505, 351, 179, and 152 from the Weather data set, the BP-Cubing algorithms and BUC are all able to compute the full cube quickly: BUC uses 0.3 seconds, whereas both BP-Cubing algorithms use 0.02 seconds, as shown in Fig. 9d.

This set of experiments has shown that top-down multiway aggregation using G-trees is a superb aggregation strategy, and the experimental results suggest that treebased aggregation is generally more efficient than recursive partition-based aggregation. Our experiments also confirmed the observation in [4] that partition-based bottomup aggregation is suitable for computing sparse data cubes.

Even though BP-Cubing(BU) and BUC both use the same bottom-up strategy, BP-Cubing(BU) has better performance than BUC when dealing with antimonotone constraints. This implies that aggregation on G-trees is a superior strategy. In Fig. 9c, we see that initially, BP-Cubing(TD) is more efficient than BP-Cubing(BU) for computing the full cube on Weather. However, for constraints with the threshold $\alpha \geq 5$, BP-Cubing(BU) outperforms BP-Cubing(TD). This may have happened because the size of the G-tree is large for sparse data, and the top-down aggregation strategy may have aggregated many groups, which turns out to fail the constraint.

\subsection{BP-Cubing versus DnA on " $\operatorname{Avg}(X)$ in $\left[\delta_{1}, \delta_{2}\right]$ ”}

In this set of experiments, we compare the performance of the BP-Cubing algorithms against DnA on the nonantimonotone constraint " $\operatorname{Avg}(X)$ in $\left[\delta_{1}, \delta_{2}\right]$. ."

Fig. 10 shows that the BP-Cubing algorithms scale very well when the $\left[\delta_{1}, \delta_{2}\right]$ range threshold becomes looser. In all data sets, the BP-Cubing algorithms show modest linear increase in computation time. In contrast, the performance of DnA degrades significantly (which may be due to the fact that its pruning is at the tuple record level: When many groups need to be processed, the search cost for the minimal partition to approximate a group becomes high (see Section 9 for more discussions)).

Figs. 10a and 10b show that in the dense data sets Census and TPC-R, the BP-Cubing algorithms significantly outperform DnA, and the BP-Cubing algorithm shows the best performance. For the constraint "Avg (X) in [500, $50,000]^{\prime \prime}$ over Census, DnA finishes in 65.04 seconds, whereas BP-Cubing(TD) finishes in only 3.53 seconds, which is 18.42 times faster. At the lower end, for the constraint "Avg (X) in [500, 10,000]," BP-Cubing(TD) is 7.09 times faster. In TPC-R, BP-Cubing(TD) outperforms DnA by 4.62-6.22 times. BP-Cubing(BU) also outperforms DnA overall, especially for larger constraint ranges. For the constraint "Avg (X) in [500, 50,000]" over Census, $\mathrm{BP}-\mathrm{Cubing}(\mathrm{BU})$ is 2.33 times faster than DnA.

Fig. 10c shows that in the sparse Weather data set, BP-Cubing(BU) achieves more significant efficiency improvement over DnA than BP-Cubing(TD). In this data set, 


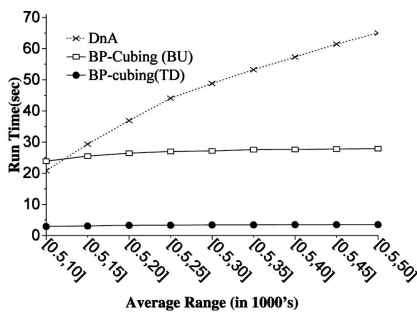

(a)

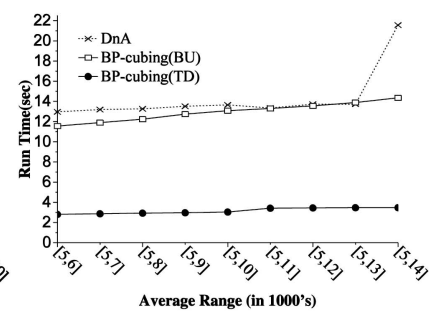

(b)

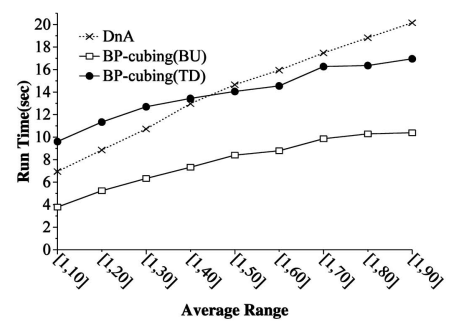

(c)

Fig. 10. BP-Cubing versus DnA on the " $\operatorname{Avg}(\mathrm{X})$ in $\left[\delta_{1}, \delta_{2}\right]$ " constraint. (a) Census. (b) TPC-R. (c) Weather.

the figure may suggest that the improvement of the BPCubing algorithms over DnA is not as pronounced as in Census and TPC-R. However, Fig. 9 has shown that in the sparse Weather data, the partition-based aggregation strategy of DnA is more efficient than the tree-based aggregation strategy of BP-Cubing. With an aggregation strategy that works not that well, the BP-Cubing algorithms still achieve better performance than DnA. Such dramatic result can only be attributed to the effectiveness of bound pruning.

As experiments have shown that BP-Cubing(TD) has better performance than BP-Cubing(BU) in general, $\mathrm{BP}-\mathrm{Cubing}(\mathrm{TD})$ is used in later experiments for comparing with DnA.

\subsection{BP-Cubing versus DnA on " $\operatorname{Var}(X) \geq \alpha$ "}

In this section, we compare our bounding techniques against DnA on the constraint " $\operatorname{Var}(\mathrm{X}) \geq \alpha$," which involves the complex nonmonotone function Var. For BPCubing, we obtain the upper bound for Var by following Table 6. For DnA, we derive the upper bound for Var as follows ([14, Example 4.2]):

$$
\begin{aligned}
\frac{\operatorname{QSUM}(X)}{\operatorname{Count} 1(c)} & -\left(\frac{\operatorname{psum} 1(c)}{\operatorname{Count} 2(X)}\right)^{2}-\left(\frac{\operatorname{nsum} 1(c)}{\operatorname{Count} 3(X)}\right)^{2} \\
& +2 \times \frac{\operatorname{psum} 2(X) \times \operatorname{nsum~} 2(X)}{(\text { Count } 4(c))^{2}}
\end{aligned}
$$

whose notations are explained later in Section 9.

The runtime of both BP-Cubing(TD) and DnA is shown in Figs. 11a, 11b, and 11c. When the Var threshold gets larger, the iceberg cubes get smaller, and both algorithms finish faster. BP-Cubing is always faster than DnA at all Var thresholds. The speedup is usually around several times. This can be attributed to the tighter bounds obtained using MSPs.

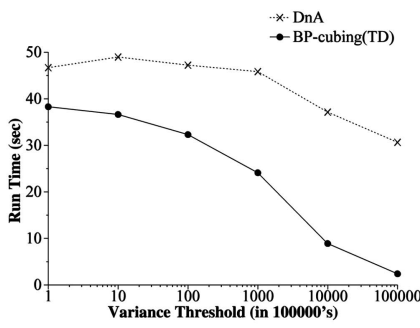

(a)

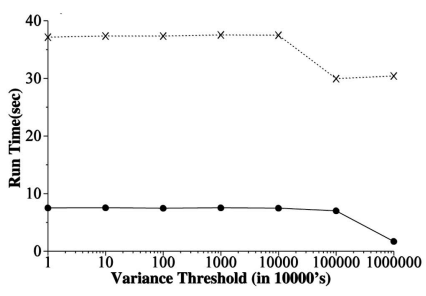

(b)

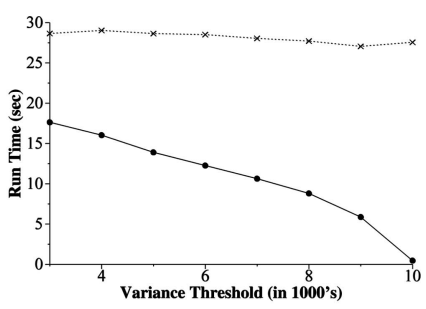

(c)

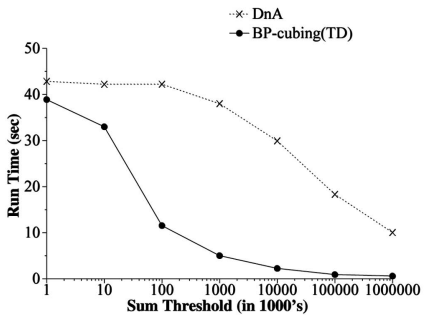

(d)

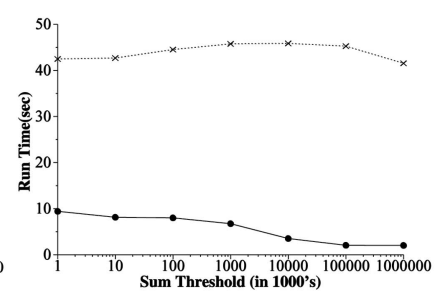

(e)

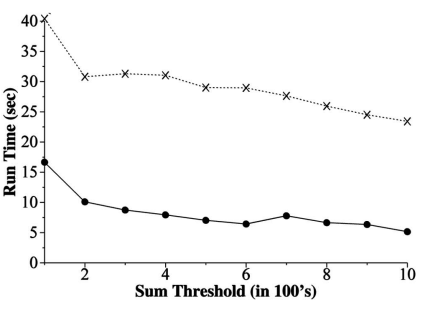

(f)
Fig. 11. BP-Cubing versus $D n A$ on " $\operatorname{Var}(X) \geq \alpha$ " and " $\operatorname{Sum}(X) \geq \beta$." (a) $\operatorname{Var}(X) \geq \alpha$ : Census. (b) $\operatorname{Var}(X) \geq \alpha$ : TPC-R. (c) $\operatorname{Var}(X) \geq \alpha$ : Weather. (d) $\operatorname{Sum}(X) \geq \beta$ : Census. (e) $\operatorname{Sum}(X) \geq \beta$ : TPC-R. (f) $\operatorname{Sum}(X) \geq \beta$ : Weather.

\subsection{BP-Cubing versus DnA on " $\operatorname{Sum}(X) \geq \beta$ "}

We now compare BP-Cubing with DnA on computing iceberg cubes defined by "Sum $(X) \geq \beta$." As shown in Table 2, $\operatorname{Sum}(X)$ is a representative for our general bounding theory, which does not involve any optimization. The upper bound of $\operatorname{Sum}(X)$ needs to be computed for pruning. In BP-Cubing, this is computed following Table 2. In DnA, this is computed following [15] (see Section 9). The artificial data sets TPC-R and Weather contain only positive measure values, which renders $\operatorname{Sum}(X) \geq \beta$ an antimonotone constraint. We randomly negated 10 percent of the measure values to make the constraint nonantimonotone so that pruning techniques can be sensibly compared. Figs. 11d, 11e, and $11 \mathrm{f}$ demonstrate the runtime of both algorithms on the three data sets under different values of $\beta$. BP-Cubing(TD) significantly outperforms DnA at all thresholds for $\operatorname{Sum}(X)$.

To ascertain the contribution of pruning toward the efficiency gain of BP-Cubing over DnA, for each algorithm, we collected the number of groups that are examined for pruning, namely, groups whose aggregates are bounded (approximated) and tested against the given constraint. There are two types of examined groups: a "true positive" (or nonsolution group) is a group whose approximated aggregate and its real aggregate pass the constraint, and a 
TABLE 8

Number of Examined Groups versus Number of Solution Groups on $\operatorname{Sum}(X) \geq \beta$

\begin{tabular}{rrr|rrr|rrr}
\hline \multicolumn{3}{c|}{ Census $\left(\beta=10^{3} . .10^{8}\right)$} & \multicolumn{3}{c|}{ TPC-R $\left(\beta=10^{4} . .10^{9}\right)$} & \multicolumn{3}{c}{ Weather $(\beta=400 . .900)$} \\
\hline Solution & BPC $^{*}$ & DnA & Solution & BPC & DnA & Solution & BPC $^{*}$ & DnA \\
\hline $19,619,534$ & $20,405,004$ & $21,378,725$ & $4,068,317$ & $4,265,176$ & $4,316,109$ & 406,628 & $1,830,753$ & $8,027,054$ \\
$14,782,195$ & $16,324,797$ & $18,609,867$ & $2,962,327$ & $3,872,446$ & $4,293,431$ & 297,216 & $1,526,206$ & $7,379,501$ \\
$3,736,088$ & $5,662,047$ & $10,087,280$ & 775,860 & $1,829,161$ & $3,690,935$ & 232,948 & $1,274,132$ & $6,695,759$ \\
644,205 & $1,432,753$ & $4,994,413$ & 102,951 & 244,951 & $1,707,564$ & 185,441 & $1,059,187$ & $6,041,456$ \\
72,459 & 233,714 & $2,195,618$ & 8,593 & 32,227 & $1,158,332$ & 148,660 & 852,296 & $5,271,776$ \\
4,137 & 20,246 & 658,320 & 357 & 3,383 & $1,014,269$ & 119,401 & 659,192 & $4,438,490$ \\
\hline
\end{tabular}

*Bound-prune cubing denotes (BP-Cubing(TD)).

"false positive" (or solution group) is a group whose approximated aggregate passes the constraint, but whose real aggregate fails the constraint.

Table 8 shows the number of examined groups versus the number of solution groups for the three data sets. The three columns list, respectively, the number of solution groups in the output and the number of groups examined in BP-Cubing(TD) and DnA. Substantially fewer groups are examined in BP-Cubing(TD) at all thresholds. This shows that BP-Cubing with MSPs has a clear advantage. Observe that, when the number of solution groups decreases, the numbers of groups examined by both algorithms decrease as well, but that number in DnA decreases at a much slower rate than that in BP-Cubing(TD).

\section{Related Work}

DnA [14], [15] is a recent approach for pruning with nonantimonotone aggregation constraints in iceberg cubing. The main idea of DnA is to divide a partition of tuples into two subspaces of positive and negative measure values, respectively, so that a given constraint can be rewritten using antimonotone or monotone constraints in subspaces. For example, consider a measure $X$, which can be positive or negative. Given the constraint $\operatorname{Avg}(\mathrm{X}) \geq \alpha$, the authors would first rewrite the original aggregate into

$$
\operatorname{Avg}(X)=\operatorname{psum}(X) / \operatorname{Count}_{1}(X)-\operatorname{nsum}(X) / \operatorname{Count}_{2}(X),
$$

where psum and nsum are the (absolute) sum of positive/ negative $X$ values, and Count ${ }_{1}$ and Count $_{2}$ are rewriting Count in the positive and negative spaces. Then, they would use $\operatorname{psum}(X) / \operatorname{Count}_{1}(c)-\operatorname{nsum}(c) / \operatorname{Count}_{2}(X)$ as a weaker antimonotone approximator for $\operatorname{Avg}(X)$, where $c$ is some smallest subpartition of the given partition. If the approximate fails the threshold $\alpha$, then all groups that are subgroups of $X$ and supergroups of $c$ are pruned.

BP-Cubing differs from DnA in several aspects:

1. Rather than the separately monotone rewriting strategy of DnA, in BP-Cubing, rewriting follows the principles that an algebraic aggregate function can be expressed as an algebraic expression of distributive aggregate functions and that the aggregate value for a group can be computed from the aggregates of MSPs. For example, given measure $X$ with MSPs $X_{1}, \ldots, X_{n}, \operatorname{Avg}(X)$ is rewritten into

$$
\begin{aligned}
& \operatorname{Sum}(X) / \operatorname{Count}(X)= \\
& \operatorname{Sum}_{i}\left(\operatorname{Sum}\left(X_{i}\right)\right) / \operatorname{Sum}_{i}\left(\operatorname{count}\left(X_{i}\right)\right) .
\end{aligned}
$$

2. In BP-Cubing, the optimization for complex aggregate functions such as Avg and Var can produce very tight bounds. Continuing with the previous example, by following Table 6, the optimized upper bound for $\operatorname{Avg}(X)$ is $\operatorname{Max}\left(\left\{\operatorname{Avg}\left(c_{i}\right)\right\}\right)$, where $c_{i}$ iterates over the smallest partitions. As $\operatorname{Avg}\left(c_{i}\right)$ is the real aggregate of a group in the search space, $\operatorname{Max}\left(\left\{\operatorname{Avg}\left(c_{i}\right)\right\}\right)$ is the optimal approximator and is tighter (smaller) than

$$
\operatorname{psum}(X) / \operatorname{Count}_{1}(c)-\operatorname{Sum}(c) / \operatorname{Count}_{2}(X),
$$

which is the bound derived by DnA.

3. MSPs are nonempty groups of tuples and BP-Cubing prunes groups. Moreover, the G-tree structure of BPCubing greatly facilitates top-down multiway aggregation and saves computation, especially for dense data. DnA calculates aggregate bounds from tuples and prunes tuples-the bottom-up recursive partitioning aggregation strategy can incur extra cost searching for and pruning tuples that do not occur in any groups that satisfy a given constraint.

4. Extra processing is needed in DnA to incorporate antimonotone constraints such as "Count $(*) \geq n . "$ Continuing with the previous example, consider the constraint " $\operatorname{Avg}(X) \geq \alpha$ and $\operatorname{Count}(*) \geq n$ " and the (ab) partition with $c, d$, and e as further partitioning dimension values. In DnA, with recursive partitioning, even if (e) is infrequent, subpartitions of (ab) and (e), namely, (abe), (abce), (abde), and (abcde), are not pruned (the Rollback tree was proposed to prune these groups). Note that these are only some of the subpartitions of $(e)$ that should be pruned. In BP-Cubing, as discussed in Section 7.5, all subpartitions of a partition failing an antimonotone constraint are pruned.

The top- $k$ average technique [7] was designed specifically for the constraint " $\operatorname{Avg}(x) \geq \alpha$ and $\operatorname{Count}(*) \geq k$ " and is not a general pruning technique for iceberg cubing. The tree-based bottom-up aggregation strategy was first proposed in [7]. Top-down aggregation was proposed independently in [16] for performing multiway aggregation in cubing; however, the work was focused on how to incorporate bottom-up pruning for antimonotone aggregation constraints into top-down aggregation. The BUC algorithm was proposed in [4], where pruning with antimonotone constraints was performed in the bottom-up recursive partitioning aggregation framework.

Our study is also related to constraint data mining [2], [3], [8], [9], [12], [13]. Agrawal and Srikant [2] wrote the first paper on pruning with the antimonotone constraint Count $(*) \geq \alpha$ for association mining. In other works on 
association mining, pruning strategies with specific constraints were proposed, namely, minimum improvement constraints [3], succinct constraints [8], convertible constraints [9], item constraints [12], and support constraints [13]. These constraints are orthogonal to the aggregation constraints that we consider in iceberg cubing.

\section{Conclusions}

In computing iceberg cubes, pruning with nonantimonotone aggregation constraints has been a challenging problem. In this paper, we have proposed pruning with nonantimonotone constraints by estimating their upper and lower bounds on a data cube from aggregates of the most specific (or minimal) partitions. We have proposed iceberg cubing algorithms, called BP-Cubing, which incorporate bounding and pruning, and incorporated top-down and bottom-up aggregation strategies by using the G-tree structure. Our extensive experiments on real and artificial data sets have shown that BP-Cubing is effective for pruning under various data characteristics, and our iceberg cubing algorithms significantly outperform state-of-the-art iceberg cubing algorithms.

\section{ACKNOWLEDGMENTS}

The authors thank the reviewers for their helpful comments. A significantly abridged version of this paper appeared in [5].

\section{References}

[1] S. Agarwal, R. Agrawal, P. Deshpande, A. Gupta, J. Naughton, R. Ramakrishnan, and S. Sarawagi, "On the Computation of Multidimensional Aggregates," Proc. Int'l Conf. Very Large Data Bases (VLDB '96), pp. 506-521, 1996.

[2] R. Agrawal and R. Srikant, "Fast Algorithms for Mining Association Rules," Proc. Int'l Conf. Very Large Data Bases (VLDB '94), pp. 487-499, 1994.

[3] R.J. Bayardo, R. Agrawal, and D. Gunopulos, "Constraint-Based Rule Mining in Large Dense Databases," Proc. 15th IEEE Int'l Conf. Data Eng. (ICDE '99), 1999.

[4] K. Beyer and R. Ramakrishnan, "Bottom-Up Computation of Sparse and Iceberg Cubes," Proc. ACM Int'l Conf. Management of Data (SIGMOD '99), 1999.

[5] L. Chou and X. Zhang, "Computing Complex Iceberg Cubes by Multiway Aggregation and Bounding," Proc. Sixth Int'l Conf. Data Warehousing and Knowledge Discovery (DaWak'04), 2004.

[6] J. Gray, S. Chaudhuri, A. Bosworth, A. Layman, D. Reichart, and M. Venkatrao, "Data Cube: A Relational Aggregation Operator Generalizing Group-By, Cross-Tab and Subtotals," Data Mining and Knowledge Discovery, vol. 1, no. 1, pp. 29-53, 1997.

[7] J. Han, J. Pei, G. Dong, and K. Wang, "Efficient Computation of Iceberg Cubes with Complex Measures," Proc. ACM Int'l Conf. Management of Data (SIGMOD '01), 2001.

[8] R. Ng, L.V.S. Lakshmanan, J. Han, and A. Pang, "Exploratory Mining and Pruning Optimizations of Constrained Associations Rules," Proc. ACM Int'l Conf. Management of Data (SIGMOD '98), pp. 13-24, 1998.

[9] J. Pei, J. Han, and L.V.S. Lakshmanan, "Mining Frequent Itemsets with Convertible Constraints," Proc. 17th IEEE Int'l Conf. Data Eng. (ICDE'01), pp. 433-442, 2001.

[10] K.A. Ross and D. Srivastava, "Fast Computation of Sparse Data Cubes," Proc. Int'l Conf. Very Large Data Bases (VLDB '97), pp. 116$125,1997$.

[11] S. Sarawagi, R. Agrawal, and R. Gupta, "On Computing the Data Cube," Technical Report RJ10026, IBM Almaden Research Center, 1996.

[12] R. Srikant, Q. Vu, and R. Agrawal, "Mining Association Rules with Item Constraints," Proc. Third Int'l Conf. Knowledge Discovery and Data Mining (KDD '97), pp. 67-73, 1997.
[13] K. Wang, Y. He, and J. Han, "Pushing Support Constraints into Frequent Itemset Mining," Proc. Int'l Conf. Very Large Data Bases (VLDB '00), 2000.

[14] K. Wang, Y. Jiang, J.X. Yu, G. Dong, and J. Han, "Pushing Aggregate Constraints by Divide-and-Approximate," Proc. 19th IEEE Int'l Conf. Data Eng. (ICDE '03), 2003.

[15] K. Wang, Y. Jiang, J.X. Yu, G. Dong, and J. Han, “Divide-andApproximate: A Novel Constraint Push Strategy for Iceberg Cube Mining," IEEE Trans. Knowledge and Data Eng., vol. 17, no. 3, pp. 354-368, 2005.

[16] D. Xin, J. Han, X. Li, and B.W. Wah, "Star-Cubing: Computing Iceberg Cubes by Top-Down and Bottom-Up Integration," Proc. Int'l Conf. Very Large Data Bases (VLDB '03), 2003.

[17] Y. Zhao, P. Deshpande, and J.F. Naughton, "An Array-Based Algorithm for Simultaneous Multidimensional Aggregates," Proc. ACM Int'l Conf. Management of Data (SIGMOD '97), 1997.

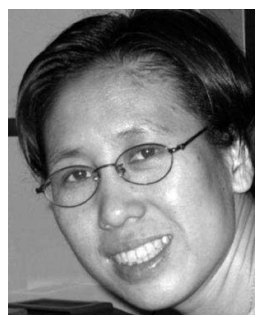

Xiuzhen Zhang received the $\mathrm{PhD}$ degree from the University of Melbourne. She is currently a senior lecturer in the School of Computer Science and Information Technology, RMIT University. Her research interests include database technology, data mining, machine learning, and their applications in new areas. She is a member of the ACM.

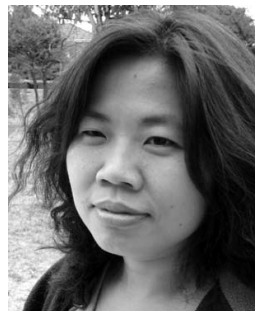

Pauline Lienhua Chou received the PhD degree from RMIT University. She currently works for the Australian government as a data analyst. Her research interests include database technology and data mining.

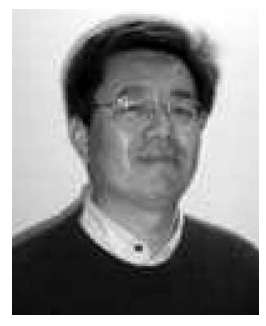

Guozhu Dong received the PhD degree from the University of Southern California in 1988. $\mathrm{He}$ is a professor at Wright State University. He previously taught at the University of Melbourne and Flinders University. He has served on numerous program committees including the IEEE International Conference on Data Engineering (ICDE), the IEEE International Conference on Data Mining (ICDM), the International Conference on Database Theory (ICDT), the ACM Symposium on Principles of Database Systems (PODS), the ACM SIGKDD International Conference on Knowledge Discovery and Data Mining, and the International Conference on Very Large Data Bases (VLDB). He was a program committee cochair of the 2003 International Conference on Web-Age Information Management (WAIM), is on the steering committee of the same conference series, is on the editorial board of the International Journal of Information Technology, and will serve as a program committee cochair of the 2007 Joint International Conference of APWeb and WAIM. His main research interests are databases, data mining, and bioinformatics. His research has been funded by government agencies such as the US National Science Foundation (NSF), the Australian Research Council (ARC), and the US Air Force Research Laboratory (AFRL) and private corporations. He has published more than 100 articles in journals and conferences, holds three US patents, and was a recipient of the IEEE ICDM 2005 Best Paper Award. He is a senior member of the IEEE and member of the ACM.

$\triangleright$ For more information on this or any other computing topic, please visit our Digital Library at www.computer.org/publications/dlib. 